\title{
Does Smartphone Addiction Fall on a Continuum of Addictive Behaviors?
}

\author{
Sheila $\mathrm{Yu}^{1, *(1)}$ and Steve Sussman ${ }^{1,2}$ \\ 1 Department of Preventive Medicine, University of Southern California, 2001 N Soto St., \\ Los Angeles, CA 90032, USA; ssussma@usc.edu \\ 2 Department of Psychology, University of Southern California, 3620 South McClintock Ave, \\ Los Angeles, CA 90089, USA \\ * Correspondence: sheilayu@usc.edu
}

Received: 7 November 2019; Accepted: 4 January 2020; Published: 8 January 2020

\begin{abstract}
Due to the high accessibility and mobility of smartphones, widespread and pervasive smartphone use has become the social norm, exposing users to various health and other risk factors. There is, however, a debate on whether addiction to smartphone use is a valid behavioral addiction that is distinct from similar conditions, such as Internet and gaming addiction. The goal of this review is to gather and integrate up-to-date research on measures of smartphone addiction (SA) and problematic smartphone use (PSU) to better understand (a) if they are distinct from other addictions that merely use the smartphone as a medium, and (b) how the disorder(s) may fall on a continuum of addictive behaviors that at some point could be considered an addiction. A systematic literature search adapted from the Preferred Reporting Items for Systematic Reviews and Meta-Analyses (PRISMA) method was conducted to find all relevant articles on SA and PSU published between 2017 and 2019. A total of 108 articles were included in the current review. Most studies neither distinguished SA from other technological addictions nor clarified whether SA was an addiction to the actual smartphone device or to the features that the device offers. Most studies also did not directly base their research on a theory to explain the etiologic origins or causal pathways of SA and its associations. Suggestions are made regarding how to address SA as an emerging behavioral addiction.
\end{abstract}

Keywords: smartphone addiction; problematic smartphone use

\section{Introduction}

After the iPhone entered global markets in 2007, significant technological advancements for smartphones have been made [1]. Smartphones offer a myriad of information sources at the touch of one's fingertips and enhance productivity (e.g., synced calendars, email, alerts and alarms, global positioning system (GPS) maps), as well as provide instant access to entertainment and social networking sites and social media channels (e.g., Facebook, Twitter, Instagram) [1,2]. Due to the high accessibility and mobility of smartphones, widespread and pervasive smartphone use has become the social norm [2], which has resulted in an increase in potential distractions that expose users to various health and other risk factors (e.g., distracted driving or walking, resulting in traffic accidents; physical health detriments, such as neck and shoulder issues, blurred vision, and wrist pain; sleep disturbances; highly sedentary habits; poor academic performance; mental health concerns; financial burdens) [1-5].

One other consequence of excessive smartphone use is the possibility of developing an addiction. The concept of addiction historically has focused on recreational drug use, exhibiting classic characteristics related to repetitive use (i.e., tolerance, withdrawal symptoms [4,5], dependence, social problems [5], and loss of control [4]). Addiction now includes behaviors in addition to substances [1-5], such as gambling disorder and Internet gambling disorder [4], as discussed in 
the latest fifth edition of the Diagnostic and Statistical Manual of Mental Disorders (DSM-5) [4-6] and in the proposed International Classification of Diseases (ICD)-11 for Mortality and Morbidity Statistics [7]. The recognition of these conditions, however, did not occur immediately, as evidence of neurobiological and psychological mechanisms indicating the addictive nature of these behavioral disorders, and accurate assessment of them, took time [8].

The acknowledgement of PSU [3] has led to a debate on whether the concept of SA is a true addiction and, if it is, if it is distinct from other technological addictions engaged in on the smartphone, such as Internet and gaming addiction. Due to the recency of this phenomenon, there has been a paucity of research on its assessment [4,5]. Assessment of SA should involve reliable instruments (e.g., good internal consistency), and demonstrate adequate content validity as a measure of addiction (attempts at appetitive need fulfillment, preoccupation, loss of control, undesired consequences [9]). In addition, such a measure should capture unique consequences of SA (e.g., use while in social situations that interfere with a flow of conversation or upsets a speaker, use while driving). Limitations of knowledge in this new arena of SA/PSU studies include self-report bias from participants, lack of standardized criteria to measure SA/PSU, and different contexts that need to be considered, which may impose variation in external demands for smartphone use (e.g., sociocultural, professional, social, and academic conflicts) [10].

While there are a variety of limitations, the growing empirical research generally seems to support the concept of SA as a genuine addictive disorder, despite the available data being insufficient in establishing a valid and definitive conceptualization of SA [4]. We propose that SA may fall along a continuum of dysregulated behavior, which is problematic and even incapacitating at some point $[8,11]$. It is important to explore how authors to date have characterized excessive smartphone misuse and understand how various factors gleaned from current research coalesce to discern a clearer picture of this emerging behavioral addiction.

\section{Research Aims}

The goal of this review was to examine the most recent studies of SA/PSU to see the extent to which there appears to be unique features of SA/PSU and how studies assess this behavior, to arrive at an understanding regarding at which point there is likely to be consensus on the condition being an addiction along a spectrum of problematic behaviors.

We also explored proneness to SA/PSU as a function of gender, as some researchers have suggested that SA may be more prevalent among females [12-23]. Other research has suggested that subsets of SA (e.g., social media addiction versus gaming addiction) may differentiate males from females in their smartphone use $[12,14,15,24-36]$.

Additionally, we examined variation as a function of age. Some researchers have suggested that SA may be more prevalent among youth and young adults as compared to older adults and elderly who use smartphones less often than youth $[12,13,19,26,30,32,34,35,37-55]$.

\section{Materials and Methods}

We conducted a systematic review of the literature on and related to SA/PSU utilizing the Preferred Reporting Items for Systematic Reviews and Meta-Analyses (PRISMA) statement [56] as a general guide. Electronic databases utilized included: MEDLINE/PubMed, ProQuest, GALE, Web of Science, Directory of Open Access Journals, Elsevier's collection, Taylor \& Francis Online, Wiley Online Library, SpringerLink, and Sage Journal's collection.

\subsection{Inclusion Criteria}

We utilized the following search terms: "(smartphone OR "mobile phone") AND (addiction OR "problematic use")", published in peer-reviewed journals between 2017 and 2019. Examining Google Scholar, we noticed a steep influx of SA/PSU articles published beginning 2017 (from 22 appearing in 2007; 41 in 2011; 164 in 2013; and 1020 in 2017). Therefore, we began the search from 2017 since this 
would best capture the current state of research in the area of SA/PSU research. Of the 170 articles returned from the search, 100 were retained for the current review after screening their titles and abstracts. The references of these articles were also searched, of which eight articles were deemed relevant and were included, allowing us to review 108 articles in total.

\subsection{Exclusion Criteria}

Articles published in a language other than English, duplicate articles (primary articles were retained), studies that did not substantively extend a parent study (i.e., only adapted established $\mathrm{SA} / \mathrm{PSU}$ scales to a new language with some validation testing), and irrelevant articles (determined after looking through the abstracts or text; examples of irrelevant studies included those assessing the efficacy of an app serving as an intervention, focusing on the risk factors of SA/PSU rather than $\mathrm{SA} / \mathrm{PSU}$ itself, and measuring invariance among SA-related scales) were excluded from the review pool (see Figure 1).

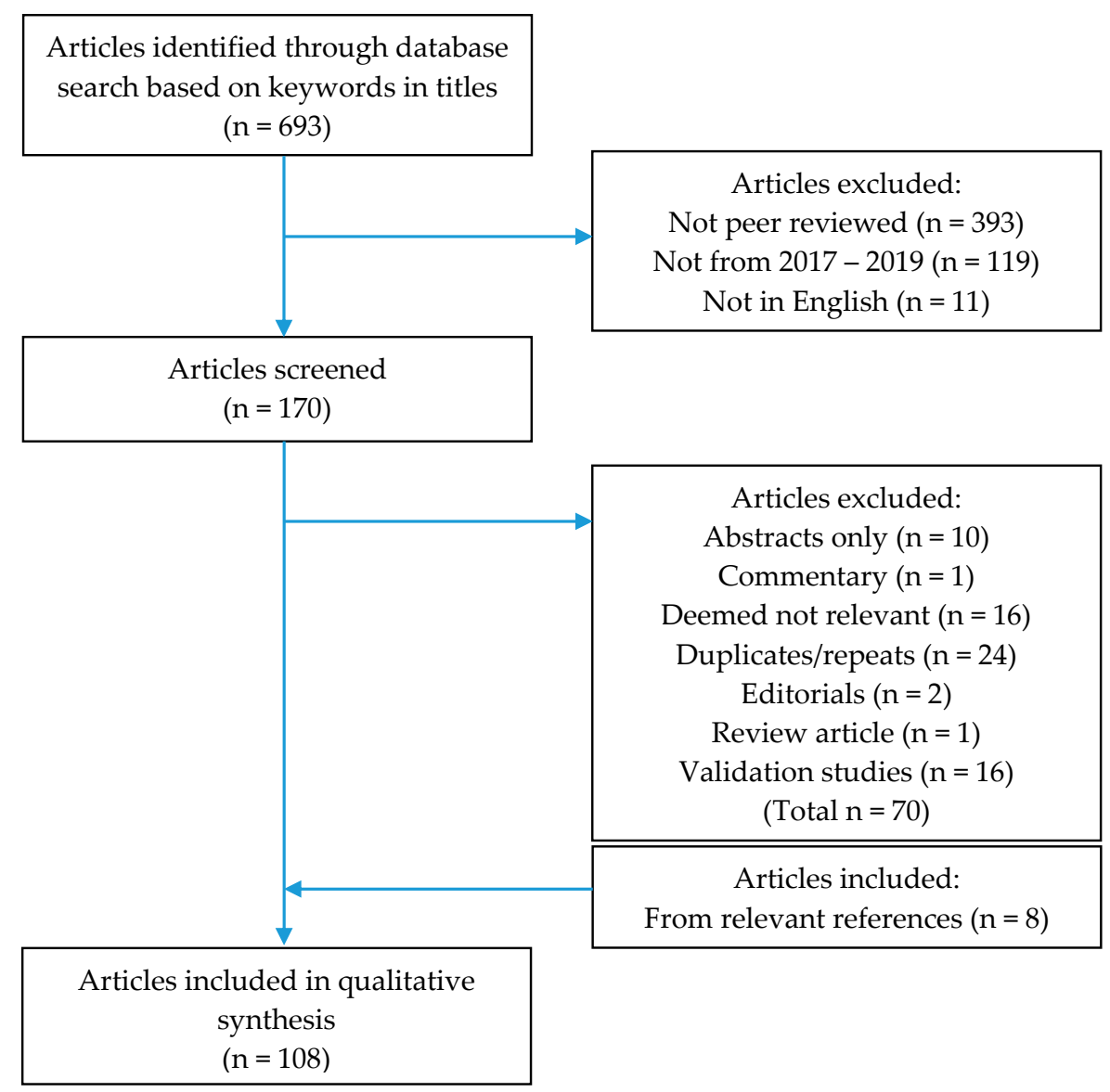

Figure 1. Process of final article selection for the current review.

\subsection{Information Synthesis}

Since most of the literature on smartphone use were based upon SA and PSU scales, we created a table to compile scales used to measure SA/PSU of smartphones, examining the following (based on Bianchi and Phillips, 2008 [3]): (1) Scale/measurement items, (2) Cronbach's alpha coefficients, and (3) variables and/or factors measured to assess smartphone use. A total of 14 relevant assessments emerged from a review of previous SA/PSU studies (see Table 1). 
Table 1. Smartphone addiction/problematic smartphone use assessments in the literature.

\begin{tabular}{|c|c|c|c|}
\hline Title (Abbreviation): Authors & $\begin{array}{l}\text { Year } \\
\text { Cat }^{1}\end{array}$ & $\begin{array}{c}\text { Items } \\
\mathrm{CA}^{2}\end{array}$ & Measures/Factors \\
\hline $\begin{array}{l}\text { Mobile Phone Problem Use Scale (MPPUS): } \\
\text { Bianchi and Phillips }\end{array}$ & $\begin{array}{l}2005 \\
\text { PSU }\end{array}$ & $\begin{array}{c}27 \\
0.93\end{array}$ & $\begin{array}{l}\text { 1. Tolerance } \\
\text { 2. Escape from other problems } \\
\text { 3. Withdrawal } \\
\text { 4. Craving } \\
\text { 5. Negative life consequences }\end{array}$ \\
\hline $\begin{array}{l}\text { Mobile Phone Addiction Index (MPAI): } \\
\text { Leung }\end{array}$ & $\begin{array}{c}2008 \\
\text { SA }\end{array}$ & $\begin{array}{l}17 \\
0.87\end{array}$ & $\begin{array}{l}\text { 1. Inability to control craving } \\
\text { 2. Feeling anxious and lost } \\
\text { 3. Withdrawal or escape } \\
\text { 4. Productivity loss }\end{array}$ \\
\hline $\begin{array}{l}\text { Problematic Mobile Phone Use Questionnaire } \\
\text { (PMPUQ): } \\
\text { Billieux, Van der Linden, and Rochat }\end{array}$ & $\begin{array}{l}2008 \\
\text { PSU }\end{array}$ & $\begin{array}{c}30 \\
0.77\end{array}$ & $\begin{array}{l}\text { 1. Dangerous/prohibited use } \\
\text { 2. Dependence } \\
\text { 3. Financial problems }\end{array}$ \\
\hline $\begin{array}{l}\text { Smart Mobile Phone Addiction Scale (MPAS): } \\
\text { Hong, Chiu, and Huang }\end{array}$ & 2012 & $\begin{array}{c}11 \\
0.90\end{array}$ & $\begin{array}{l}\text { 1. Academic problems and effects } \\
\text { 2. Time management and problems } \\
\text { 3. Substitute satisfaction }\end{array}$ \\
\hline $\begin{array}{c}\text { Problematic Use of Mobile Phones (PUMP) Scale: } \\
\text { Merlo, Stone, and Bibbey }\end{array}$ & $\begin{array}{l}2013 \\
\text { PSU }\end{array}$ & $\begin{array}{c}20 \\
0.94\end{array}$ & $\begin{array}{l}\text { 1. Tolerance } \\
\text { 2. Withdrawal } \\
\text { 3. Longer time than intended } \\
\text { 4. Great deal of time spent } \\
\text { 5. Craving } \\
\text { 6. Activities given up or reduced } \\
\text { 7. Use despite physical/psychological problems } \\
\text { 8. Failure to fulfill role obligations } \\
\text { 9. Use in physically hazardous situations } \\
\text { 10. Use despite social/interpersonal problems }\end{array}$ \\
\hline $\begin{array}{l}\text { Smartphone Addiction Scale (SAS): } \\
\text { Kwon, Lee, Won, Park, Min, Hahn, Gu, Choi, } \\
\text { and Kim }\end{array}$ & $\begin{array}{c}2013 \\
\text { SA }\end{array}$ & $\begin{array}{l}48 \\
0.97\end{array}$ & $\begin{array}{l}\text { 1. Daily-life disturbance } \\
\text { 2. Positive anticipation } \\
\text { 3. Withdrawal } \\
\text { 4. Cyberspace-oriented relationship } \\
\text { 5. Overuse } \\
\text { 6. Tolerance }\end{array}$ \\
\hline $\begin{array}{l}\text { Short Version for Adolescents } \\
\text { (SAS-SV): } \\
\text { Kwon, Kim, Cho, Yang }\end{array}$ & $\begin{array}{c}2013 \\
\text { SA }\end{array}$ & $\begin{array}{c}10 \\
0.91\end{array}$ & $\begin{array}{l}\text { 1. Daily-life disturbance } \\
\text { 2. Withdrawal } \\
\text { 3. Cyberspace-oriented relationship } \\
\text { 4. Overuse } \\
\text { 5. Tolerance }\end{array}$ \\
\hline $\begin{array}{l}\text { Smartphone Addiction Scale for College Students } \\
\text { (SAS-C): } \\
\text { Su, Pan, Liu, Chen, Wang, and Li }\end{array}$ & $\begin{array}{c}2014 \\
\text { SA }\end{array}$ & $\begin{array}{c}22 \\
0.93\end{array}$ & $\begin{array}{l}\text { 1. Withdrawal behavior } \\
\text { 2. Salience behavior } \\
\text { 3. Social comfort } \\
\text { 4. Negative effects } \\
\text { 5. Use of application (app) } \\
\text { 6. Renewal of app }\end{array}$ \\
\hline $\begin{array}{c}\text { Smartphone Addiction Management System } \\
\text { (SAMS): } \\
\text { Lee, Ahn, Choi, and Choi }\end{array}$ & $\begin{array}{c}2014 \\
\text { SA }\end{array}$ & $\begin{array}{c}14 \\
\mathrm{~N} / \mathrm{A}\end{array}$ & $\begin{array}{l}\text { 1. Daily-life disturbance } \\
\text { 2. Virtual world orientation } \\
\text { 3. Withdrawal } \\
\text { 4. Tolerance }\end{array}$ \\
\hline $\begin{array}{l}\text { Smartphone Addiction Inventory (SPAI): } \\
\text { Lin, Chang, Lee, Tseng, Kuo, and Chen }\end{array}$ & $\begin{array}{c}2014 \\
\text { SA }\end{array}$ & $\begin{array}{c}26 \\
0.94\end{array}$ & $\begin{array}{l}\text { 1. Compulsive behavior } \\
\text { 2. Functional impairment } \\
\text { 3. Withdrawal } \\
\text { 4. Tolerance }\end{array}$ \\
\hline $\begin{array}{l}\text { Korean Smartphone Addiction Proneness Scale } \\
\text { (SAPS }{ }^{3} \text { ): } \\
\text { Kim, Lee, Lee, Nam, and Chung }\end{array}$ & $\begin{array}{c}2014 \\
\text { SA }\end{array}$ & $\begin{array}{c}29 \\
0.88\end{array}$ & $\begin{array}{l}\text { 1. Disturbance of adaptive functions } \\
\text { 2. Withdrawal } \\
\text { 3. Tolerance } \\
\text { 4. Virtual life orientation }\end{array}$ \\
\hline $\begin{array}{l}\text { Smartphone Addiction Scale (SPAS): } \\
\text { Bian and Leung }\end{array}$ & $\begin{array}{c}2014 \\
\text { SA }\end{array}$ & $\begin{array}{c}19 \\
0.83\end{array}$ & $\begin{array}{l}\text { 1. Disregard of harmful consequences } \\
\text { 2. Preoccupation } \\
\text { 3. Inability to control craving } \\
\text { 4. Productivity loss } \\
\text { 5. Feeling anxious and lost }\end{array}$ \\
\hline $\begin{array}{l}\text { Mobile Phone Problem Use Scale: derivation of a } \\
\text { short scale (MPPUS-10): } \\
\text { Foerster, Roser, Schoeni, and Röösli }\end{array}$ & $\begin{array}{l}2015 \\
\text { PSU }\end{array}$ & $\begin{array}{c}10 \\
0.85\end{array}$ & $\begin{array}{l}\text { 1. Craving } \\
\text { 2. Withdrawal } \\
\text { 3. Dependence } \\
\text { 4. Loss of control } \\
\text { 5. Negative life consequences }\end{array}$ \\
\hline $\begin{array}{c}\text { Short form of Smartphone Addiction Inventory } \\
\text { (SPAI-SF): } \\
\text { Lin, Pan, Lin, and Chen }\end{array}$ & 2016 & $\begin{array}{c}10 \\
0.84\end{array}$ & $\begin{array}{l}\text { 1. Compulsive behavior } \\
\text { 2. Functional impairment } \\
\text { 3. Withdrawal } \\
\text { 4. Tolerance }\end{array}$ \\
\hline
\end{tabular}

Notes. ${ }^{1}$ Category: Smartphone addiction (SA) or problematic smartphone use (PSU); ${ }^{2}$ Cronbach's alpha value; ${ }^{3}$ First developed by Korea's National Information Society Agency (2011) "Report on the Development of Korean Smartphone Addiction Proneness Scale for Youth and Adults" (also known as S-Scale) [Korean] 
We compared the items across these assessments to see which were common among the scales (see Table 2). Based on Griffiths' (2005) six-component model of addiction, we categorized the measured items based on the components argued to be needed "to be present for a behavior to be operationally defined as addictive": Salience (activity becomes most important, dominating aspect), mood modification (subjective experience of feeling better, whether calming down and/or feeling less destressed), tolerance (more activity needed to feel activity's previous effect), withdrawal (abrupt termination of activity results in unpleasant feeling, psychologically and/or physiologically), conflict (activity causes conflict between addict and others or ones' self-image), and relapse (addictive activity patterns recur) [57].

Table 2. Comparison of major validated scale variables based on Griffiths' component model.

\begin{tabular}{lcccccccccc}
\hline & MPPUS & MPAI & MPAS & SAS & SAS-SV & SPAI & SPAI-SF & SAPS & SPAS & Total \\
\hline Conflict & $\sqrt{ }$ & $\sqrt{ }$ & $\sqrt{ }$ & $\sqrt{ }$ & $\sqrt{ }$ & $\sqrt{ }$ & $\sqrt{ }$ & $\sqrt{ }$ & $\sqrt{ }$ & 9 \\
Withdrawal & $\sqrt{ }$ & $\sqrt{ }$ & $\sqrt{ }$ & $\sqrt{ }$ & $\sqrt{ }$ & $\sqrt{ }$ & $\sqrt{ }$ & $\sqrt{ }$ & $\sqrt{ }$ & 9 \\
Tolerance & $\sqrt{ }$ & $\sqrt{ }$ & $\sqrt{ }$ & $\sqrt{ }$ & $\sqrt{ }$ & $\sqrt{ }$ & $\sqrt{ }$ & $\sqrt{ }$ & $\sqrt{ }$ & 9 \\
Salience & $\sqrt{ }$ & $\sqrt{ }$ & $\sqrt{ }$ & $\sqrt{ }$ & $\sqrt{ }$ & $\sqrt{ }$ & $\sqrt{ }$ & $\sqrt{ }$ & $\sqrt{ }$ & 9 \\
Mood & $\sqrt{ }$ & $\sqrt{ }$ & & $\sqrt{ }$ & & $\sqrt{ }$ & & $\sqrt{ }$ & $\sqrt{ }$ & 6 \\
Relapse & & & & & & $\sqrt{ }$ & $\sqrt{ }$ & $\sqrt{ }$ & & 3 \\
\hline
\end{tabular}

We also extracted the following information from the studies (see Table 3): (1) First author; (2) year published; (3) whether the study classified smartphone use as an addiction (SA) or problematic smartphone use (PSU); (4) sample size (n); (5) the scale used to assess SA/PSU; (6) if SA/PSU was clearly defined; (7) if studies mentioned the negative attributes and/or risk factors of SA/PSU; (8) if studies distinguished SA/PSU from other technological addictions (e.g., Internet and gaming addiction); (9) if studies differentiated if the addiction is to the actual phone device or to what the phone device offers, such as functions, services, and/or content (e.g., social networking services, apps, etc.); and (10) if studies were structured around a theory or model to explain SA/PSU and how it affects the smartphone user. Under the scale column "adapted", scales refer to scales adapted from SA/PSU assessment scales used in previous studies (e.g., authors compiled $x$ items from scale $A$ and $y$ items from scale $B$ to create their own scale, $S$ ) and/or input from field experts.

Table 3. Characteristics of relevant smartphone studies, 2017-2019.

\begin{tabular}{|c|c|c|c|c|c|c|c|}
\hline First Author & Year & Cat $^{1}$ & Scale & Define $^{2}$ & Distinct $^{3}$ & D v. $F^{4}$ & Theory \\
\hline Abed & 2018 & SA & Adapted & No & No & No & $\mathrm{n} / \mathrm{a}$ \\
\hline Akodu & 2018 & SA & SAS-SV & No & No & Yes & $\mathrm{n} / \mathrm{a}$ \\
\hline Akturk & 2018 & SA & SAS-SV & Yes & No & No & $\mathrm{n} / \mathrm{a}$ \\
\hline AlAbdulwahab & 2017 & SA & SAS & No & No & No & $\mathrm{n} / \mathrm{a}$ \\
\hline Alavi & 2018 & SA & MPPUS & No & No & No & Yes 5 \\
\hline Albursan & 2019 & SA & Adapted & No & Yes & Yes & $\mathrm{n} / \mathrm{a}$ \\
\hline Alhassan & 2018 & SA & SAS-SV & Yes & No & No & $\mathrm{n} / \mathrm{a}$ \\
\hline Alhazmi & 2018 & SA & SAS-SV & Yes & No & No & $\mathrm{n} / \mathrm{a}$ \\
\hline Arefin & 2017 & SA & Adapted & Yes & Yes & Yes & $\mathrm{n} / \mathrm{a}$ \\
\hline Arnavut & 2018 & SA & SAS-SV & Yes & No & No & $\mathrm{n} / \mathrm{a}$ \\
\hline Barnes & 2019 & SA & Adapted & Yes & Yes & Yes & Yes 6 \\
\hline Basu & 2018 & SA & Adapted & Yes & No & No & $\mathrm{n} / \mathrm{a}$ \\
\hline Beison & 2017 & PSU & MPPUS & No & No & No & Yes $^{7}$ \\
\hline Cerit & 2018 & SA & SAS & Yes & No & No & $\mathrm{n} / \mathrm{a}$ \\
\hline Cha & 2018 & SA & SAPS & Yes & No & No & $\mathrm{n} / \mathrm{a}$ \\
\hline Chang & 2019 & SA & SPAI & Yes & No & No & $\mathrm{n} / \mathrm{a}$ \\
\hline Chen & 2018 & SA & Adapted & No & Yes & Yes & Yes $^{8}$ \\
\hline Chen & 2017 & SA & Adapted & Yes & No & No & Yes $^{9}$ \\
\hline Chen & 2017 & SA & SAS-SV & Yes & No & No & $\mathrm{n} / \mathrm{a}$ \\
\hline Chiang & 2019 & SA & SPAI-SF & No & No & No & $\mathrm{n} / \mathrm{a}$ \\
\hline Cho & 2017 & SA & SAPS & Yes & No & No & Yes ${ }^{10}$ \\
\hline
\end{tabular}


Table 3. Cont.

\begin{tabular}{|c|c|c|c|c|c|c|c|}
\hline First Author & Year & Cat $^{1}$ & Scale & Define $^{2}$ & Distinct $^{3}$ & D v. $F^{4}$ & Theory \\
\hline Cho & 2017 & SA & $\mathrm{AMT}^{11}$ & No & No & No & $\mathrm{n} / \mathrm{a}$ \\
\hline Choi & 2017 & SA & SAS & Yes & No & No & $\mathrm{n} / \mathrm{a}$ \\
\hline Chou & 2019 & SA & SPAI-SF & No & No & No & Yes $^{12}$ \\
\hline Chung & 2018 & SA & SAPS & No & No & No & $\mathrm{n} / \mathrm{a}$ \\
\hline Cocoradă & 2018 & SA & SAS-SV & Yes & No & No & Yes $^{13}$ \\
\hline Contractor & 2017 & PSU & SAS-SV & Yes & No & No & Yes ${ }^{14}$ \\
\hline De-Sola & 2017 & SA & MPPUS & Yes & Yes & No & $\mathrm{n} / \mathrm{a}$ \\
\hline Ding & 2019 & PSU & SOCS & No & No & Yes & $\mathrm{n} / \mathrm{a}$ \\
\hline Duke & 2017 & SA & SAS-SV & No & Yes & Yes & $\mathrm{n} / \mathrm{a}$ \\
\hline Elhai & 2017 & PSU & SAS & No & No & No & Yes 15 \\
\hline Elserty & 2018 & SA & SAS-SV & Yes & No & No & $\mathrm{n} / \mathrm{a}$ \\
\hline Gao & 2018 & SA & MPAI & Yes & No & No & Yes $^{16}$ \\
\hline Gao & 2017 & SA & MPAS & No & No & No & $\mathrm{n} / \mathrm{a}$ \\
\hline Gezgin & 2018 & SA & SAS-SV & Yes & No & No & $\mathrm{n} / \mathrm{a}$ \\
\hline Gligor & 2019 & SA & $\mathrm{MPDQ}^{17}$ & No & No & No & $\mathrm{n} / \mathrm{a}$ \\
\hline Gökçearslan & 2018 & SA & SAS-SV & Yes & No & No & $\mathrm{n} / \mathrm{a}$ \\
\hline Habibi & 2018 & SA & Adapted & Yes & No & No & $\mathrm{n} / \mathrm{a}$ \\
\hline Han & 2017 & SA & MPAI & Yes & Yes & No & Yes $^{18}$ \\
\hline Нао & 2019 & SA & MPAI & Yes & No & No & $\mathrm{n} / \mathrm{a}$ \\
\hline Hawi & 2017 & SA & SAS-SV & No & No & No & $\mathrm{n} / \mathrm{a}$ \\
\hline Heo & 2018 & SA & SAPS & No & No & No & $\mathrm{n} / \mathrm{a}$ \\
\hline Herrero & 2019 & SA & SPAS & No & No & No & Yes ${ }^{10}$ \\
\hline Herrero & 2019 & SA & SPAS & Yes & No & No & $\mathrm{n} / \mathrm{a}$ \\
\hline Herrero & 2019 & SA & SPAS & Yes & No & No & $\mathrm{n} / \mathrm{a}$ \\
\hline Ihm & 2018 & SA & Adapted & No & No & No & $\mathrm{n} / \mathrm{a}$ \\
\hline Jeong & 2019 & SA & SAPS & Yes & Yes & No & $\mathrm{n} / \mathrm{a}$ \\
\hline Jin & 2017 & SA & SAS-SV & Yes & No & No & Yes ${ }^{18,19}$ \\
\hline Jo & 2018 & SA & SAPS & No & No & No & $\mathrm{n} / \mathrm{a}$ \\
\hline Khoury & 2019 & SA & SPAI & No & Yes & No & $\mathrm{n} / \mathrm{a}$ \\
\hline Kim & 2018 & PSU & Adapted & Yes & No & No & Yes 20 \\
\hline Kim & 2017 & PSU & Adapted & Yes & No & No & $\mathrm{n} / \mathrm{a}$ \\
\hline Kim & 2019 & SA & SAPS & No & No & No & $\mathrm{n} / \mathrm{a}$ \\
\hline Kim & 2018 & SA & SAPS & Yes & No & No & $\mathrm{n} / \mathrm{a}$ \\
\hline Kim & 2017 & SA & SAPS & No & No & No & Yes $^{18}$ \\
\hline Kim & 2017 & SA & SAPS & Yes & No & No & Yes ${ }^{14}$ \\
\hline Kim & 2017 & SA & SAS & No & No & No & $\mathrm{n} / \mathrm{a}$ \\
\hline Kim & 2018 & SA & SAPS & Yes & No & No & Yes $^{18}$ \\
\hline Kita & 2018 & SA & SAS-SV & Yes & No & No & $\mathrm{n} / \mathrm{a}$ \\
\hline Konan & 2019 & SA & SAS-SV & Yes & No & No & $\mathrm{n} / \mathrm{a}$ \\
\hline Konan & 2018 & SA & SAS-SV & No & No & No & $\mathrm{n} / \mathrm{a}$ \\
\hline Kuang-Tsan & 2017 & SA & MPAS & No & No & No & $\mathrm{n} / \mathrm{a}$ \\
\hline Kumcağız & 2019 & SA & SAS-SV & No & No & No & $\mathrm{n} / \mathrm{a}$ \\
\hline Kumcağız & 2017 & SA & SAS-SV & No & No & No & $\mathrm{n} / \mathrm{a}$ \\
\hline Kuss & 2018 & PSU & PMPUQ & Yes & No & No & Yes 21 \\
\hline Kwak & 2018 & SA & Adapted & No & No & No & Yes $^{22}$ \\
\hline Kwan & 2017 & SA & SAS & Yes & No & Yes & Yes 23,24 \\
\hline Lan & 2018 & SA & MPIAS 25 & No & No & No & $\mathrm{n} / \mathrm{a}$ \\
\hline Lee & 2018 & PSU & SAPS & Yes & No & No & Yes 26 \\
\hline Lee & 2017 & SA & SAPS & Yes & Yes & No & $\mathrm{n} / \mathrm{a}$ \\
\hline Lee & 2017 & SA & SAPS & No & No & No & $\mathrm{n} / \mathrm{a}$ \\
\hline Lee & 2017 & SA & Adapted & No & No & No & $\mathrm{n} / \mathrm{a}$ \\
\hline Lee & 2018 & SA & Adapted & No & No & No & $\mathrm{n} / \mathrm{a}$ \\
\hline Lee & 2018 & SA & Adapted & No & No & No & $\mathrm{n} / \mathrm{a}$ \\
\hline Lee & 2018 & SA & SAS-SV & No & Yes & No & $\mathrm{n} / \mathrm{a}$ \\
\hline Lee & 2018 & SA & SAS-SV & Yes & Yes & No & Yes 27 \\
\hline $\mathrm{Li}$ & 2018 & SA & SPAS & No & No & No & Yes 28 \\
\hline Lian & 2018 & SA & MPAI & Yes & No & No & Yes ${ }^{26,29}$ \\
\hline Lian & 2017 & SA & MPAI & Yes & No & No & Yes ${ }^{26}$ \\
\hline Lin & 2017 & SA & App & Yes & Yes & Yes & $\mathrm{n} / \mathrm{a}$ \\
\hline Liu & 2018 & SA & MPAI & Yes & No & No & Yes $^{22}$ \\
\hline
\end{tabular}


Table 3. Cont.

\begin{tabular}{|c|c|c|c|c|c|c|c|}
\hline First Author & Year & Cat $^{1}$ & Scale & Define $^{2}$ & Distinct $^{3}$ & D v. $F^{4}$ & Theory \\
\hline Liu & 2017 & SA & MPAI & No & No & No & Yes 31 \\
\hline Mahapatra & 2018 & SA & Adapted & Yes & No & Yes & Yes $^{32}$ \\
\hline Matar B. & 2017 & SA & SPAI & No & Yes & No & $\mathrm{n} / \mathrm{a}$ \\
\hline Nayak & 2018 & SA & Adapted & Yes & Yes & No & $\mathrm{n} / \mathrm{a}$ \\
\hline Noë & 2019 & SA & SAS & Yes & Yes & Yes & $\mathrm{n} / \mathrm{a}$ \\
\hline Parasuraman & 2017 & SA & Adapted & No & No & Yes & $\mathrm{n} / \mathrm{a}$ \\
\hline Salvi & 2018 & SA & Adapted & Yes & No & No & $\mathrm{n} / \mathrm{a}$ \\
\hline Sekhon & 2018 & SA & MPPUS & Yes & No & No & $\mathrm{n} / \mathrm{a}$ \\
\hline Sun & 2019 & SA & SAS $(\mathrm{Ku})$ & Yes & No & No & Yes $^{34}$ \\
\hline Tunc-Aksan & 2019 & SA & SAS & No & No & Yes & Yes 35 \\
\hline Wang & 2018 & SA & SAS-SV & No & No & No & Yes $^{36,37}$ \\
\hline Wang & 2017 & SA & SAS-SV & No & No & No & Yes 38 \\
\hline Wolniewicz & 2018 & PSU & SAS-SV & Yes & Yes & Yes & Yes ${ }^{15,16}$ \\
\hline $\mathrm{Xu}$ & 2019 & PSU & MPAS & Yes & No & No & Yes 22,39 \\
\hline Yang & 2019 & SA & MPAI & Yes & No & No & Yes 40 \\
\hline Yang & 2019 & PSU & Adapted & Yes & No & No & $\mathrm{n} / \mathrm{a}$ \\
\hline Yildiz Durak & 2017 & SA & SAS & Yes & Yes & No & Yes 41 \\
\hline Yildiz & 2017 & SA & SAS-SV & No & No & No & $\mathrm{n} / \mathrm{a}$ \\
\hline
\end{tabular}

Notes. ${ }^{1}$ Category based on study title: Smartphone addiction (SA) or problematic smartphone use (PSU); ${ }^{2}$ Define: If SA or PSU was defined; ${ }^{3}$ Distinct: If studies distinguished SA from other technological addictions (e.g., Internet, gaming addiction); ${ }^{4} \mathrm{D}$ v. F: If studies differentiated if the addiction is to the actual phone device or to what the phone device offers, such as functions, services, and/or content (e.g., social networking services, apps, etc.); ${ }^{5}$ Psychosocial Theory of Development (Erikson, 1963) and Adolescent Identity Paradigm (Marcia, 1991); ${ }^{6}$ Cognitive absorption (Agarwal \& Karahanna, 2000); ${ }^{7}$ Reward Deficiency Syndrome hypothesis (Blum et al., 1996); ${ }^{8}$ Social influence theory (Rashotte, 2007); ${ }^{9}$ Four categories of drinking motives (Stewart \& Devine, 2000); ${ }^{10}$ Big Five personality traits (Norman, 1963); ${ }^{11}$ Addiction Measurement Tools of Measuring Smartphone Addiction of Children-Adolescents (Korea Network Information Center); ${ }^{12}$ 6-T Internet attitude model (Chou, Wu, \& Chen, 2013); ${ }^{13}$ Theory of Reasoned Action (Fishbein \& Ajzen, 1975); ${ }^{14}$ Impulsive pathway perspective (Billieux, 2012); ${ }^{15}$ Uses and Gratifications Theory (UGT) (Blumler \& Katz, 1974); ${ }^{16}$ Compensatory Internet Use Theory (CIUT) (Kardefelt-Winther, 2014); ${ }^{17}$ MPDQ: Mobile phone dependence questionnaire; ${ }^{18}$ Attachment theory (Bowlby, 1969); ${ }^{19}$ Psychoanalytic theory (Kassel et al., 2007); ${ }^{20}$ Social enhancement model (Kraut et al., 2002); ${ }^{21}$ Biopsychosocial model of addiction (Griffiths, 2005); ${ }^{22}$ General strain theory (Agnew, 1992); ${ }^{23}$ Theory of parenting style (Baumrind, 1971); ${ }^{24}$ First theory of self-regulation (Asgari et al., 2011); ${ }^{25}$ MPIAS: Mobile Phone Internet Addiction Scale; ${ }^{26}$ Problem behavior theory (PBT) (Jessor, 1977); ${ }^{27}$ Power distance belief (Hofstede, 1980); ${ }^{28}$ Media system dependency (MSD) theory (Ball-Rokeach \& DeFleur, 1976); ${ }^{29}$ Social skills deficit theory (Valkenburg \& Peter, 2007); ${ }^{30}$ Social compensation theory (Zell \& Moeller, 2018); ${ }^{31}$ Response style theory (Nolen-Hoeksema, 1991); ${ }^{32}$ Incentive-Sensitization (Robinson \& Berridge, 2003) \& Learning Theory (Wallace, 1999); ${ }^{33}$ Hierarchy of needs theory (Maslow, 1968); ${ }^{34}$ Risky families model (Repetti, 2002); ${ }^{35}$ Intrinsic motivation theory (Przybylski, Weinstein, Ryan, \& Rigby, 2009); ${ }^{36}$ Sensation seeking theory (Zuckerman, 1994); ${ }^{37}$ Social support buffering hypothesis (Cohen \& Wills, 1985); ${ }^{38}$ Cognitive-behavioral model (Davis, 2001); ${ }^{39}$ Resilience theory (Fergus \& Zimmerman, 2005); 40 Diathesis-stress theories (Monroe \& Simons, 1991) and stress-buffering hypothesis (Cohen \& Edwards, 1989); ${ }^{41}$ Social Cognitive Theory (Bandura, 1986);

42 Sociometer theory of self-esteem (Leary et al., 1995).

To supplement Table 3, we conducted an in-depth analysis on the study samples (see Table 4), specifically (1) the group population, either child/youth (in primary, elementary, middle, or high school), university student (students in college, graduate, and vocational/technical/institute schools were all categorized as university students), or adult; (2) sample size, $n$; (3) percentage of males in the sample; (4) mean age and age range of participants; (5) participant nationality; (6) sociodemographic characteristics (SDCs) (biometrics, such as body mass index (BMI), height, and weight; number of children; education; income; marital status; region or residence, such as rural or urban; and 
work/employment status); and (7) reported study biases. SDC data were reported for university students and adults, usually the parents of children.

The point of Table 4 was to determine the generalizability of the studies' results: Can the research findings and conclusions of a particular group of interest be extended to the population at large? If a particular population sample is studied across age and nationality, does that imply that that group is at-risk for SA?

Table 4. Demographic profile of smartphone study samples and reported study biases.

\begin{tabular}{|c|c|c|c|c|c|c|c|}
\hline First Author & Pop ${ }^{1}$ & $n^{2}$ & Males (\%) & Age $\left(m(r){ }^{3}\right)$ & Nationality & $\begin{array}{c}\text { SDC }^{4} \\
\text { (BCEIMRW) }\end{array}$ & Reported Biases \\
\hline Abed & Univ & 229 & 35 & $\mathrm{n} / \mathrm{a}$ & Iraqi & MRW & None \\
\hline Akodu & Univ & 77 & 57 & 22 & Nigerian & B & Sampling \\
\hline Akturk & Univ & 1156 & 49 & $\mathrm{n} / \mathrm{a}$ & Turkish & CEI & Sampling \\
\hline AlAbdulwahab & Univ & 78 & 50 & 21 & Saudi & B & None \\
\hline Alavi & Univ & 500 & 21 & $28(18-31+)$ & Iranian & E M & Sampling \\
\hline Albursan & Univ & 2008 & 45 & $22(17-28)$ & Arab & & Sampling \\
\hline Alhassan & Adult & 935 & 34 & $32(18-55+)$ & Saudi & EI $R$ & Multiple \\
\hline Alhazmi & Univ & 181 & 48 & 24 & Saudi & B $\mathrm{M}$ & Sampling \\
\hline Arefin & Univ & 247 & 54 & $(18-27)$ & Bangladeshi & & Sampling \\
\hline Arnavut & Adult & 714 & 58 & $(18-30+)$ & Turkish & & Sampling \\
\hline Barnes & Univ & 140 & 31 & $(18-35+)$ & American & $\mathrm{E}$ & Multiple \\
\hline Basu & Univ & 388 & 60 & 20 & Indian & $\mathrm{R}$ & Sampling \\
\hline Beison & Univ & 100 & 25 & $20(18-23)$ & American ${ }^{5}$ & EI & None \\
\hline Cerit & Univ & 214 & 20 & $20(18-26)$ & Turkish & & Recall \\
\hline Cha & MS & 1824 & 51 & 16 & Korean & W & Multiple \\
\hline Chang & ES/Adult & 5089 & $52 / 31$ & $\mathrm{n} / \mathrm{a} / 43$ & Taiwanese & EIM & Multiple \\
\hline Chen & Univ & 2000 & 49 & $21(17-23)$ & Taiwanese & & Multiple \\
\hline Chen & Univ & 384 & 54 & $\mathrm{n} / \mathrm{a}$ & Chinese & & Multiple \\
\hline Chen & Univ & 1441 & 48 & $20(17-26)$ & Chinese & $\mathrm{R}$ & Multiple \\
\hline Chiang & ES/MS & 2155 & 52 & $\mathrm{n} / \mathrm{a}$ & Taiwanese & EIM & Multiple \\
\hline Cho & Adult & 400 & 52 & $(20-40+)$ & Korean & E W & Multiple \\
\hline Cho & PS/Adult & 303 & $7 / 51$ & $(20-40+) /(0-6)$ & Korean & EI W & Sampling \\
\hline Choi & HS & 1020 & 52 & $\mathrm{n} / \mathrm{a}$ & Korean & $\mathrm{R}$ & Sampling \\
\hline Chou & HS/Adult & 1444 & $43 / \mathrm{n} / \mathrm{a}$ & $\mathrm{n} / \mathrm{a} / \mathrm{n} / \mathrm{a}$ & Taiwanese & EI & Recall \\
\hline Chung & MS/HS & 1796 & 46 & $15(13-18)$ & Korean & & Sampling \\
\hline Cocoradă & HS/Univ & 717 & 35 & 20 & Romanian & & Multiple \\
\hline Contractor & Adult & 346 & 42 & 34 & American 5 & IM W & Multiple \\
\hline De-Sola & Adult & 1126 & 48 & $33(16-65)$ & Spanish & E R & Multiple \\
\hline Ding & Univ & 849 & 56 & $\mathrm{n} / \mathrm{a}$ & Chinese & & Multiple \\
\hline Duke & Adult & 262 & 36 & 32 & German & & Recall \\
\hline Elhai & Adult & 308 & 54 & 33 & American 5 & EIM W & Multiple \\
\hline Elserty & Univ & 420 & 32 & 20 & Egyptian & B & None \\
\hline Gao & Univ & 1105 & 29 & $21(16-25)$ & Chinese & I & Multiple \\
\hline Gao & Univ & 722 & 48 & $20(15-24)$ & Chinese & C I R & Multiple \\
\hline Gezgin & HS & 161 & 58 & 16 & Turkish & & Multiple \\
\hline Gligor & Univ & 150 & 44 & 27 & Romanian & $\mathrm{R}$ & Multiple \\
\hline Gökçearslan & Univ & 885 & 41 & $\mathrm{n} / \mathrm{a}$ & Turkish & I & None \\
\hline Habibi & HS & 271 & $\mathrm{n} / \mathrm{a}$ & 17 & Indonesian & & None \\
\hline Han & Univ & 543 & 41 & $20(17-22)$ & Chinese & & Sampling \\
\hline Hao & Univ & 847 & 51 & $20(18-24)$ & Chinese & $\mathrm{C}$ & Sampling \\
\hline Hawi & Univ & 381 & 59 & $21(17-27)$ & Lebanese & & Multiple \\
\hline Heo & HS & 790 & 23 & $\mathrm{n} / \mathrm{a}$ & Korean & & None \\
\hline Herrero & All & 526 & 52 & $(15-55+)$ & Spanish & E $R$ & None \\
\hline Herrero & All & 241 & 55 & $(15-55+)$ & Spanish & E $R$ & Sampling \\
\hline Herrero & All & 416 & 52 & $(15-55+)$ & Spanish & E $R$ & Selection \\
\hline Ihm & Youth & 2000 & 50 & 12 & Korean & & Recall \\
\hline Jeong & HS & 768 & 58 & $\mathrm{n} / \mathrm{a}$ & Korean & IM & Multiple \\
\hline Jin & Univ & 297 & 55 & $20(17-24)$ & Chinese & I & Sampling \\
\hline Jo & All & 7003 & 45 & $(14-39)$ & Korean & R & Recall \\
\hline Khoury & Univ & 100 & 48 & $(18-25)$ & Brazilian $^{5}$ & $\mathrm{IM}$ & Response \\
\hline Kim & Adult & 615 & 51 & $30(19-40)$ & American 5 & & Recall \\
\hline Kim & All & 930 & 52 & $26(13-40)$ & American 5 & M & Recall \\
\hline Kim & MS/HS & 4512 & 45 & 15 & Korean & IM & Multiple \\
\hline Kim & Youth & 3380 & 51 & $(10-19)$ & Korean & EIMRW & Information \\
\hline Kim & Univ & 200 & 37 & $22(19-28)$ & Korean & & Sampling \\
\hline Kim & Univ & 608 & 30 & 23 & Korean & I & Multiple \\
\hline Kim & HS & 1479 & 48 & $\mathrm{n} / \mathrm{a}$ & Korean & B & Sampling \\
\hline Kim & Univ & 313 & 42 & $22(17-29)$ & Korean & & Sampling \\
\hline Kita & YA & 221 & 65 & $19(17-22)$ & Israeli & & Sampling \\
\hline
\end{tabular}


Table 4. Cont.

\begin{tabular}{|c|c|c|c|c|c|c|c|c|}
\hline First Author & Pop ${ }^{1}$ & $\mathrm{n}^{2}$ & Males (\%) & Age $\left(m(r)^{3}\right)$ & Nationality & \multicolumn{2}{|c|}{$\begin{array}{c}\text { SDC }^{4} \\
\text { (BCEIMRW) }\end{array}$} & Reported Biases \\
\hline Konan & Univ & 496 & 25 & $\mathrm{n} / \mathrm{a}$ & Turkish & & & None \\
\hline Konan & Univ & 330 & 36 & $(20-24)$ & Turkish & & & None \\
\hline Kuang-Tsan & Univ & 332 & 65 & $(18-22)$ & Taiwanese & & & Sampling \\
\hline Kumcağ1z & HS & 352 & 44 & $16(14-19)$ & Turkish & $\mathrm{E}$ & W & Multiple \\
\hline Kumcağız & Adult & 428 & 37 & $40(21-65)$ & Turkish & CEIM & $\mathrm{I} \mathrm{W}$ & Sampling \\
\hline Kuss & All & 273 & 26 & $28(16-65)$ & Various & & & None \\
\hline Kwak & MS & 1170 & 42 & $\mathrm{n} / \mathrm{a}$ & Korean & I & & Sampling \\
\hline Kwan & Univ & 211 & 35 & 22 & Hong Kong & & & None \\
\hline Lan & Univ & 1044 & 48 & 21 & Chinese & & & Information \\
\hline Lee & Youth & 231 & 40 & $16(13-18)$ & Korean & & & Sampling \\
\hline Lee & MS & 370 & 49 & 13 & Korean & & & Multiple \\
\hline Lee & MS/HS & 3000 & 53 & $\mathrm{n} / \mathrm{a}$ & Korean & & & Sampling \\
\hline Lee & MS/HS & 1125 & 51 & $\mathrm{n} / \mathrm{a}$ & Korean & EI I & $\mathrm{R}$ & Multiple \\
\hline Lee & Univ & 125 & 49 & $\mathrm{n} / \mathrm{a}$ & Korean & & & None \\
\hline Lee & Univ & 324 & 9 & $\mathrm{n} / \mathrm{a}$ & Korean & & & Multiple \\
\hline Lee & MS & 490 & 100 & 14 & Korean & $\mathrm{E}$ & & Multiple \\
\hline Lee & Adult & 778 & $63 / 58^{6}$ & $35 / 25^{6}$ & Various & EI & & Information \\
\hline $\mathrm{Li}$ & Adult & 527 & 46 & $27(18-35)$ & Chinese & EIM & I W & Sampling \\
\hline Lian & Univ & 716 & 54 & $20(18-24)$ & Chinese & & $\mathrm{R}$ & Sampling \\
\hline Lian & Univ & 682 & 58 & $19(18-24)$ & Chinese & & & Recall \\
\hline Lin & Univ & 79 & 72 & 22 & Taiwanese & & & Sampling \\
\hline Liu & HS & 899 & 46 & 17 (14-19) & Chinese & & & Sampling \\
\hline Liu & Univ & 465 & 31 & $19(16-24)$ & Chinese & & & Sampling \\
\hline Liu & HS & 1196 & 53 & $17(14-20)$ & Chinese & & & Multiple \\
\hline $\mathrm{Lu}$ & MS & 1311 & 54 & 15 & Various & & & Sampling \\
\hline Mahapatra & HS/Univ & 330 & 58 & $(15-20)$ & Indian & & & Multiple \\
\hline Matar B. & Univ & 688 & 53 & 21 & Lebanese & & W & Recall \\
\hline Megna & Adult & 52 & 46 & $27(18-35)$ & Italian & & & None \\
\hline Mei & Univ & 1034 & 47 & 20 & Chinese & C I & $\mathrm{R}$ & Multiple \\
\hline Nayak & Univ & 429 & 35 & $20(16-29)$ & Indian & & & None \\
\hline Noë & Adult & 64 & 53 & $25(19-46)$ & British & $\mathrm{E}$ & W & Sampling \\
\hline Parasuraman & Adult & 409 & 42 & $23(18-55)$ & Malaysian & $\mathrm{E}$ & W & Sampling \\
\hline Salvi & Univ & 100 & 59 & $21(18-25)$ & Indian & & & None \\
\hline Sekhon & Univ & 80 & 50 & $(20-24)$ & Indian & & & Sampling \\
\hline Serin & Univ & 287 & 14 & $\mathrm{n} / \mathrm{a}$ & Turkish & CEIM & & Multiple \\
\hline Sok & Univ & 139 & 16 & $\mathrm{n} / \mathrm{a}$ & Korean & & & Sampling \\
\hline Song & PS/Adult & 328 & $\mathrm{n} / \mathrm{a} / 0$ & $(3-5) / \mathrm{n} / \mathrm{a}$ & Korean & $\mathrm{E}$ & W & Multiple \\
\hline Sun & HS & 1041 & 56 & $12(11-15)$ & Chinese & & & Multiple \\
\hline Tunc-Aksan & HS & 296 & 54 & $\mathrm{n} / \mathrm{a}$ & Turkish & $\mathrm{E}$ & & None \\
\hline Wang & MS & 655 & 55 & $17(15-19)$ & Chinese & & & Multiple \\
\hline Wang & MS & 768 & 44 & $17(15-19)$ & Chinese & $\mathrm{E}$ & W & Multiple \\
\hline Wolniewicz & Univ & 296 & 43 & 20 & American 5 & $\mathrm{E}$ & W & Multiple \\
\hline $\mathrm{Xu}$ & MS & 316 & 47 & $14(12-16)$ & Chinese & & & Sampling \\
\hline Yang & HS & 1258 & 53 & $17(14-20)$ & Chinese & & & Sampling \\
\hline Yang & Univ & 218 & 58 & $18(16-19+)$ & Taiwanese & & & Multiple \\
\hline Yildiz Durak & MS/HS & 612 & 52 & $13(10-18)$ & Turkish & CEI & $\mathrm{R}$ & Multiple \\
\hline Yildiz & HS & 262 & 50 & $17(14-19)$ & Turkish & & & Sampling \\
\hline You & Univ & 653 & 50 & $20(17-25)$ & Chinese & EI & & Sampling \\
\hline Youn & Youth & 158 & 53 & 15 (12-19) & Korean & EI & & Multiple \\
\hline
\end{tabular}

Notes. ${ }^{1}$ Pop: Population general group (PS: Primary school; ES: Elementary school; MS: Middle school; HS: High school; Univ: University, college, or institute of technology students; YA: Young adult); ${ }^{2}$ Sample size $n ;{ }^{3} \mathrm{~m}(\mathrm{r})$ : mean age (range, if available); ${ }^{4}$ SDC: Common sociodemographic characteristics besides gender, age, and ethnicity $(\mathrm{B}=$ biometrics: height, weight, $\mathrm{BMI} ; \mathrm{C}=$ number of children; $\mathrm{E}=$ education; $\mathrm{I}=$ income; $\mathrm{M}=$ marital status; $\mathrm{R}=$ regional/residence: urban or rural; $\mathrm{W}$ : work/employment status); ${ }^{5}$ Reports racial status (e.g., African-American/Black, American Indian, Asian, Native Hawaiian/other Pacific Islander, White) and ethnicity (e.g., Hispanic/Latino, Not Hispanic/Latino); ${ }^{6}$ Of 778 total, 431 US and 347 Chinese participants: male $\%$ and mean age of US and Chinese, respectively.

To supplement Table 4 on the characteristics of the studies' samples (refer to Table 5), we examined the quality of the data collection process by noting if samples were selected as a convenience sample or randomly selected and if a sample size calculation was calculated before participant recruitment. We also categorized the data collected as consequences pertaining to: Academics (grades, academic performance), physiological (headache, eye/neck/hand pain), psychological (experiencing depression, loneliness, cravings), social (peer relationships), or usage; and whether it was objective or subjective (such as an objective clinical diagnosis of depression or a subjective self-report of experiencing depression). 
Additionally, we assessed the operationalization of theory by noting if the SA/PSU scale used in the study met Griffiths' criteria for behavioral addictions: Conflict, mood modification, relapse, salience, tolerance, and withdrawal. The 2004 Surgeon General's report, The Health Consequences of Smoking, developed a framework for interpreting evidence, specifying a four-level hierarchy for interpreting evidence: (a) Evidence is sufficient to infer a causal relationship (multiple scientifically supported evidence), (b) evidence is suggestive but not sufficient to infer a causal relationship (scientifically supported evidence), (c) evidence is inadequate to infer the presence or absence of a causal relationship (evidence that is not scientifically supported and/or is sparse, of poor quality, or conflicting), or (d) evidence is suggestive of no causal relationship (no evidence) [58]. We also noted if the assumption of addiction, referencing back to the title assumption of whether the authors viewed excessive smartphone use as SA or PSU, influenced the study results.

Table 5. The quality of samples, level of evidence, and theoretical framework of the studies.

\begin{tabular}{|c|c|c|c|c|c|c|c|}
\hline First Author & Sel $^{1}$ & SSS $^{2}$ & Evidence $^{3}$ & $\mathrm{Obj}^{4}$ & Int $^{5}$ & Griffiths $^{6}$ & Inf $^{7}$ \\
\hline Abed & $\mathrm{R}$ & No & PhPs & $S$ & I & $\mathrm{n} / \mathrm{a}$ & Yes \\
\hline Akodu & $\mathrm{C}$ & No & $\mathrm{Ph}$ & $\mathrm{O}$ & $\mathrm{Sg}$ & C STW & Yes \\
\hline Akturk & $\mathrm{C}$ & Yes & $S$ & $S$ & $\mathrm{Sg}$ & C STW & Yes \\
\hline AlAbdulwahab & $\mathrm{C}$ & Yes & $\mathrm{Ph}$ & $S$ & I & CM STW & Yes \\
\hline Alavi & $\mathrm{C}$ & Yes & Ps & $\mathrm{O}$ & I & CM STW & Yes \\
\hline Albursan & $\mathrm{R}$ & No & Ps & $S$ & I & n/a & Yes \\
\hline Alhassan & $\mathrm{R}$ & No & Ps & $S$ & I & C STW & Yes \\
\hline Alhazmi & $\mathrm{R}$ & Yes & $\mathrm{Ph}$ & $S$ & I & C STW & Yes \\
\hline Arefin & $\mathrm{C}$ & No & A & $\mathrm{O}$ & $\mathrm{Sg}$ & CM STW & Yes \\
\hline Arnavut & $\mathrm{R}$ & No & $\mathrm{U}$ & $S$ & $\mathrm{I}$ & C STW & Yes \\
\hline Barnes & $\mathrm{C}$ & No & Ps & $S$ & I & C STW & Yes \\
\hline Basu & $\mathrm{R}$ & Yes & $\mathrm{U}$ & $S$ & $\mathrm{I}$ & CM STW & Yes \\
\hline Beison & $\mathrm{n} / \mathrm{a}$ & No & S & $S$ & $\mathrm{Sg}$ & CM STW & Yes \\
\hline Cerit & $\mathrm{R}$ & Yes & $\mathrm{Ph}$ & $S$ & I & CM STW & Yes \\
\hline Cha & $\mathrm{R}$ & No & PhPsU & $S$ & I & CMRSTW & Yes \\
\hline Chang & $\mathrm{R}$ & No & SU & $S$ & I & CMRSTW & Yes \\
\hline Chen & $\mathrm{C}$ & No & PsS & $S$ & I & $\mathrm{n} / \mathrm{a}$ & PSU $^{8}$ \\
\hline Chen & $\mathrm{C}$ & No & Ps & $S$ & I & CMRSTW & Yes \\
\hline Chen & $\mathrm{R}$ & No & PhPs & $S$ & I & C STW & Yes \\
\hline Chiang & $\mathrm{R}$ & No & Ps & $S$ & I & TW & Yes \\
\hline Cho & $\mathrm{R}$ & No & Ps & $S$ & I & CMRSTW & Yes \\
\hline Cho & $\mathrm{R}$ & No & Ps & $S$ & $\mathrm{I}$ & $\mathrm{n} / \mathrm{a}$ & Yes \\
\hline Choi & $\mathrm{n} / \mathrm{a}$ & No & $\mathrm{U}$ & $S$ & I & C $\quad$ TW & Yes \\
\hline Chou & $\mathrm{R}$ & No & $\mathrm{U}$ & $S$ & I & TW & Yes \\
\hline Chung & $\mathrm{R}$ & No & $\mathrm{Ph}$ & $S$ & I & CMRSTW & Yes \\
\hline Cocoradă & $\mathrm{C}$ & No & PsU & $S$ & I & C STW & Yes \\
\hline Contractor & $\mathrm{C}$ & No & Ps & $S$ & I & C STW & Yes \\
\hline De-Sola & $\mathrm{C}$ & No & Ps & $S$ & I & CM STW & Yes \\
\hline Ding & $\mathrm{R}$ & No & PsU & $S$ & I & CMRST & Yes \\
\hline Duke & $\mathrm{C}$ & No & $\mathrm{U}$ & $S$ & I & C STW & Yes \\
\hline Elhai & $\mathrm{C}$ & No & $\mathrm{PsU}$ & $S$ & $\mathrm{I}$ & CM STW & Yes \\
\hline Elserty & $\mathrm{C}$ & No & $\mathrm{PhU}$ & $S$ & I & C STW & Yes \\
\hline Gao & $\mathrm{C}$ & No & Ps & $S$ & $\mathrm{I}$ & CM STW & Yes \\
\hline Gao & $\mathrm{C}$ & No & Ps & $S$ & I & C RSTW & Yes \\
\hline Gezgin & C & No & PsU & $S$ & I & C STW & Yes \\
\hline Gligor & $\mathrm{n} / \mathrm{a}$ & No & Ps & $S$ & I & CM STW & Yes \\
\hline Gökçearslan & $\mathrm{C}$ & No & PsS & $S$ & I & C STW & Yes \\
\hline Habibi & $\mathrm{n} / \mathrm{a}$ & No & Ps & $S$ & I & C STW & Yes \\
\hline Han & $\mathrm{R}$ & No & Ps & $S$ & I & CM STW & Yes \\
\hline Нао & $\mathrm{R}$ & No & PsU & $S$ & I & CM STW & Yes \\
\hline Hawi & $\mathrm{R}$ & No & Ps & $S$ & I & C STW & Yes \\
\hline Heo & $\mathrm{C}$ & Yes & Ps & $S$ & I & CMRSTW & Yes \\
\hline Herrero & $\mathrm{R}$ & No & PsSU & $S$ & I & CM STW & Yes \\
\hline Herrero & $\mathrm{R}$ & No & Ps & $S$ & I & CM STW & Yes \\
\hline Herrero & $\mathrm{R}$ & No & Ps & $S$ & I & CM STW & Yes \\
\hline Ihm & $\mathrm{R}$ & No & $S$ & $S$ & $\mathrm{I}$ & CMRSTW & Yes \\
\hline Jeong & $\mathrm{n} / \mathrm{a}$ & No & PsS & $S$ & I & CMRSTW & Yes \\
\hline Jin & $\mathrm{C}$ & No & Ps & PsS & $\mathrm{I}$ & C STW & Yes \\
\hline Jo & $\mathrm{n} / \mathrm{a}$ & No & Ps & S & I & CMRSTW & Yes \\
\hline Khoury & $\mathrm{R}$ & No & $\mathrm{Ph}$ & $\mathrm{O}$ & $\mathrm{Sg}$ & CMRSTW & Yes \\
\hline
\end{tabular}


Table 5. Cont.

\begin{tabular}{|c|c|c|c|c|c|c|c|}
\hline First Author & Sel $^{1}$ & SSS $^{2}$ & Evidence $^{3}$ & $\mathrm{Obj}^{4}$ & Int $^{5}$ & Griffiths ${ }^{6}$ & $\operatorname{Inf}^{7}$ \\
\hline Kim & $\mathrm{R}$ & No & Ps & $S$ & I & STW & Yes \\
\hline Kim & $\mathrm{R}$ & No & Ps & S & I & STW & Yes \\
\hline Kim & $\mathrm{R}$ & No & Ps & S & I & CMRSTW & Yes \\
\hline Kim & $\mathrm{R}$ & No & $S$ & S & I & CMRSTW & Yes \\
\hline Kim & $\mathrm{n} / \mathrm{a}$ & Yes & Ps & S & I & CMRSTW & Yes \\
\hline Kim & $\mathrm{C}$ & No & $\mathrm{Ph}$ & $S$ & $\mathrm{Sg}$ & CMRSTW & Yes \\
\hline Kim & $\mathrm{n} / \mathrm{a}$ & No & $\mathrm{Ph}$ & S & $\mathrm{I}$ & CM STW & Yes \\
\hline Kim & $\mathrm{C}$ & No & Ps & S & I & CMRSTW & Yes \\
\hline Kita & $\mathrm{C}$ & No & $S$ & $\mathrm{O}$ & $\mathrm{Sg}$ & STW & Yes \\
\hline Konan & $\mathrm{R}$ & No & PsS & $S$ & I & C STW & Yes \\
\hline Konan & $\mathrm{C}$ & No & Ps & S & I & C STW & Yes \\
\hline Kuang-Tsan & $\mathrm{C}$ & No & $S$ & S & $\mathrm{Sg}$ & C RSTW & Yes \\
\hline Kumcağ1z & $\mathrm{C}$ & No & S & S & I & CM STW & Yes \\
\hline Kumcağız & $\mathrm{C}$ & No & S & S & I & C STW & Yes \\
\hline Kuss & $\mathrm{C}$ & No & Ps & S & I & C RSTW & Yes \\
\hline Kwak & $\mathrm{C}$ & No & PsS & $S$ & $\mathrm{Sg}$ & CMRSTW & Yes \\
\hline Kwan & $\mathrm{C}$ & No & PsS & S & I & CM STW & Yes \\
\hline Lan & $\mathrm{R}$ & No & Ps & S & $\mathrm{Sg}$ & $\mathrm{n} / \mathrm{a}$ & Yes \\
\hline Lee & $\mathrm{C}$ & Yes & PsS & S & $\mathrm{Sg}$ & CMRSTW & Yes \\
\hline Lee & $\mathrm{C}$ & No & Ps & $\mathrm{O}$ & $\mathrm{Sg}$ & CMRSTW & PSU $^{8}$ \\
\hline Lee & $\mathrm{n} / \mathrm{a}$ & No & $S$ & $S$ & Sg & CMRSTW & Yes \\
\hline Lee & $\mathrm{R}$ & No & $\mathrm{Ph}$ & S & I & C STW & Yes \\
\hline Lee & $\mathrm{R}$ & No & $S$ & $\mathrm{O}$ & $\mathrm{Sg}$ & CMRSTW & Yes \\
\hline Lee & $\mathrm{C}$ & No & $S$ & $S$ & I & CM STW & Yes \\
\hline Lee & $\mathrm{n} / \mathrm{a}$ & No & Ps & S & I & C STW & Yes \\
\hline Lee & $\mathrm{n} / \mathrm{a}$ & No & Ps & S & I & C STW & Yes \\
\hline $\mathrm{Li}$ & $\mathrm{C}$ & No & S & S & I & CM STW & Yes \\
\hline Lian & $\mathrm{C}$ & No & S & $S$ & I & CM STW & Yes \\
\hline Lian & $\mathrm{C}$ & No & S & S & I & CM STW & Yes \\
\hline Lin & $\mathrm{n} / \mathrm{a}$ & No & $\mathrm{U}$ & $\mathrm{O}$ & $\mathrm{Sg}$ & $\mathrm{n} / \mathrm{a}$ & Yes \\
\hline Liu & $\mathrm{R}$ & No & Ps & $S$ & I & CM STW & Yes \\
\hline Liu & $\mathrm{C}$ & No & PsS & $S$ & I & C STW & Yes \\
\hline Liu & $\mathrm{C}$ & No & PhPs & S & I & CM STW & Yes \\
\hline $\mathrm{Lu}$ & $\mathrm{C}$ & No & S & S & I & C RSTW & Yes \\
\hline Mahapatra & $\mathrm{C}$ & No & APsS & S & I & C STW & Yes \\
\hline Matar B. & $\mathrm{R}$ & No & PsU & S & I & CMRSTW & Yes \\
\hline Megna & $\mathrm{C}$ & No & $\mathrm{Ph}$ & $\mathrm{O}$ & $\mathrm{Sg}$ & C STW & Yes \\
\hline Mei & $\mathrm{C}$ & Yes & Ps & $S$ & I & CM STW & Yes \\
\hline Nayak & $\mathrm{R}$ & No & $\mathrm{AU}$ & $S$ & I & C STW & Yes \\
\hline Noë & $\mathrm{C}$ & No & $\mathrm{U}$ & $\mathrm{O}$ & $\mathrm{Sg}$ & CM STW & Yes \\
\hline Parasuraman & $\mathrm{C}$ & No & $\mathrm{U}$ & $S$ & I & $\mathrm{n} / \mathrm{a}$ & Yes \\
\hline Salvi & $\mathrm{n} / \mathrm{a}$ & No & $\mathrm{Ph}$ & $\mathrm{O}$ & $\mathrm{Sg}$ & $\mathrm{n} / \mathrm{a}$ & Yes \\
\hline Sekhon & $\mathrm{n} / \mathrm{a}$ & No & Ps & $S$ & I & CM STW & Yes \\
\hline Serin & $\mathrm{C}$ & No & Ps & $S$ & I & C STW & Yes \\
\hline Sok & $\mathrm{C}$ & Yes & PsSU & $S$ & I & $\mathrm{n} / \mathrm{a}$ & Yes \\
\hline Song & $\mathrm{C}$ & No & S & S & I & CMRSTW & Yes \\
\hline Sun & $\mathrm{n} / \mathrm{a}$ & No & PsS & $S$ & I & $\mathrm{n} / \mathrm{a}$ & Yes \\
\hline Tunc-Aksan & $\mathrm{R}$ & No & APsS & $S$ & I & CM STW & Yes \\
\hline Wang & $\mathrm{n} / \mathrm{a}$ & No & PsS & $S$ & I & STW & Yes \\
\hline Wang & $\mathrm{n} / \mathrm{a}$ & No & PsS & $S$ & I & STW & Yes \\
\hline Wolniewicz & $\mathrm{C}$ & No & PsU & $S$ & I & STW & Yes \\
\hline $\mathrm{Xu}$ & $\mathrm{n} / \mathrm{a}$ & No & APs & $S$ & I & C RSTW & Yes \\
\hline Yang & $\mathrm{R}$ & No & Ps & $S$ & I & CM STW & Yes \\
\hline Yang & $\mathrm{C}$ & No & PhPsU & $S$ & I & C $\quad S W$ & Yes \\
\hline Yildiz Durak & $\mathrm{C}$ & No & Ps & $S$ & I & CM STW & Yes \\
\hline Yildiz & $\mathrm{C}$ & No & Ps & $S$ & I & CSTW & Yes \\
\hline You & $\mathrm{C}$ & No & Ps & $S$ & I & C RSTW & Yes \\
\hline Youn & $\mathrm{C}$ & No & PsS & $S$ & I & CM STW & Yes \\
\hline
\end{tabular}

Notes. ${ }^{1}$ Sel: Selection of sample: $\mathrm{C}=$ convenience sample, $\mathrm{R}=$ randomly selected; ${ }^{2}$ SSS: Sufficiency of sample size: Yes or No; ${ }^{3}$ Evidence: Type of data presented: $\mathrm{A}=$ academic, $\mathrm{Ph}=$ physiological, $\mathrm{Ps}=$ psychological, $\mathrm{S}=$ social, $\mathrm{U}=$ usage; ${ }^{4}$ Obj: Objectivity: $\mathrm{O}=$ objective, $\mathrm{S}=$ subjective; ${ }^{5} \mathrm{IE}$ : Interpretation of the level of evidence: $\mathrm{Sf}=$ sufficient, $\mathrm{Sg}=$ suggestive but not sufficient, $\mathrm{I}=$ inadequate, $\mathrm{NR}=$ suggestive of no causal relationship; ${ }^{6} \mathrm{Griffiths:} \mathrm{C}=\mathrm{conflict}$, $\mathrm{M}=$ mood modification, $\mathrm{R}=$ relapse, $\mathrm{S}=$ salience, $\mathrm{T}=$ tolerance, $\mathrm{W}=$ withdrawal; ${ }^{7}$ Inf: Assumption of addiction influencing study results: Yes or No; ${ }^{8}$ Was titled an SA study but conclusions use PSU terminology. 


\section{Results}

\subsection{The Scales Used to Assess SA/PSU and Their Internal Consistency}

The majority of the studies in the current review (96 out of 108) fell under the SA category (89\%). Excluding one study that did not use a scale, there were nine major scales used among the studies, in order of frequency: Smartphone Addiction Scale Short Version (SAS-SV) (26\%), Smartphone Addiction Proneness Scale (SAPS) (13\%), Smartphone Addiction Scale (SAS) (9\%), Mobile Phone Addiction Index (MPAI) (8\%), Mobile Phone Addiction Scale (MPAS) (5\%), Mobile Phone Problem Use Scale (MPPUS) (4\%), Smartphone Addiction Scale (SPAS) (4\%), Smartphone Addiction Inventory (SPAI) (3\%), and SPAI Short Form (SPAI-SF) (2\%). Overall, most studies either used SAS or SAS-SV $(35 \%)$ or some adapted form of an SA/PSU-assessment scale based on scales from previous studies and/or input from field experts (26\%). Excluding the adapted scale studies, 8 of the 12 PSU studies $(67 \%)$ used SA scales to assess problematic use instead of a PSU scale, and 3 of the 95 SA studies (3\%) used PSU scales to assess SA instead of SA scales (refer to Table 3).

In terms of meeting Griffiths' criteria for behavioral addictions, all of the major scales met conflict, withdrawal, tolerance, and salience. Six of the nine met mood modification and only three met relapse (refer to Table 2). Excluding nine studies in which specific adapted scale items were unavailable, one study that did not use a scale, and 79 that used one of the nine major scales, the remaining 19 studies used an adapted SA/PSU scale that met at least half of Griffiths' criteria for behavioral addictions (mean of 4.79 , SD of 0.92 ): One study (5\% of 19 ) met three criteria, seven (37\%) met four, six (32\%) met five, and five $(26 \%)$ met all six.

All scales demonstrated validity: All scales provided a Cronbach's alpha (CA) value as a measure of internal consistency, and performed factor structure analyses, ensuring that factor fit properly explains correlations among outcomes $[59,60]$. Other evaluations of validity also included testing the concurrent validity of the scale with other established scales. The CA of the nine major scales ranged from 0.83 to 0.97 , with a mean of 0.90 and standard deviation (SD) of 0.05 (refer to Table 1 ).

\subsection{How Studies Defined SA/PSU}

In total, 87 of the 108 studies (81\%) attempted a working definition of SA/PSU, of which 56 explicitly stated the definition in the paper (64\%), while the rest simply characterized SA/PSU by its negative risk factors or listed associated traits. Nearly all of the studies (96\%) mentioned the negative risk factors associated with SA/PSU, including physical (insomnia, pain in neck and wrists, eye soreness, etc.) and physiological concerns (depression, anxiety, loneliness, etc.). SA has been defined as a maladaptive dependency on and/or obsessive-compulsive use of the smartphone device [18], a state of being immersed in uncontrollable smartphone usage [41], and the inability to properly regulate smartphone usage to the point of experiencing adverse consequences in one's daily life (Billieux, 2012) [21]. Interestingly, PSU has been similarly defined as "an excessive or uncontrolled use of smartphone" (also Billieux, 2012) [61]. It is interesting to note that the same reference has been used to define both SA and PSU, highlighting the interchangeability of the two terms.

Overall, 64 out of 108 studies (59\%) mentioned other technological addictions, such as Internet and gaming addiction, in their paper. Of the 64 studies, only $13(20 \%)$ explicitly argued that SA is separate and distinct from Internet addiction (2 out of 4 PSU studies; 11 out of 47 SA studies). In total, 20 studies out of 108 (19\%) (4 out of 13 PSU studies; 16 out of 95 SA studies) mentioned whether SA is an addiction to the actual smartphone device or to the functions/services/content that the smartphone offers users, such as social networking services and various apps that are usually not found on other devices (refer to Table 3).

\subsection{Theories Adopted Across These Studies}

More than half of the studies (63\%) did not explicitly base their study upon a specific established theory (refer to Table 3). Nine of 12 (75\%) PSU studies used a theory (five of nine, 42\%) or a concept, 
model, or hypothesis (four of nine, 33\%) to guide their research; while 31 of 96 SA studies (29\%) used a theory ( 23 of $96,24 \%$ ) or a concept or model (eight of $96,8 \%$ ) to guide their study (refer to Table 3 ).

The following four theories in the current review stood out as guiding points on understanding SA and how its pathways may work. First, social influence theory (Kelman,1974) helps to illustrate how socially influential factors predict a user's intended and actual behavior in virtual settings, which is affected by three social processes: "Compliance (normative influence from others' expectations), internalization (congruence of one's goals with others' goals), and identification (conception of one's self in terms of the group's defining features)" [39]. This theory could help to explain SA among impressionable youths, who are more prone to peer pressure to what their peers may deem cool, which could include engaging in addictive behaviors on the smartphone (e.g., games, social media, streaming videos on Twitch), as well as among adults whose lifestyles are affected by today's heavily connected environment, which makes smartphone use a social norm.

The second theory, the theory of reasoned action (Fishbein \& Ajzen, 1975), explains that a person's actual behavior is determined by their intention to behave in a certain way, being influenced both by their own attitudes and the social context. The attitudes towards the smartphone (positive, negative, fear of missing out, and task switching) were considered key predictors of addiction [62]. This perspective focuses on the intrapersonal reasons a person may fall into SA, with positive attitudes potentially bringing about positive reinforcement to keep engaging in the cycle of excessive smartphone use. Other theories helping to illustrate user adoption and acceptance of information technologies include diffusion of innovations (Rogers, 1962), the theory of planned behavior (Ajzen, 1985), and the technology acceptance model (TAM) (Davis, 1989) [25].

However, the previous theories are limited in explaining how attitudes, perceptions, and beliefs are shaped around information technologies. To address this conceptual shortfall, cognitive absorption (CA) (Agarwal \& Karahanna, 2000) was proposed as a motivating factor of usage behavior through "cognitive complexity beliefs". CA was defined as "a state of deep involvement with software ... where highly engaging and engrossing experiences result in users' 'deep attention' and complete immersion and engagement with an activity" [25]. The multidimensional construct of CA has five dimensions: Temporal dissociation, focused immersion, heightened enjoyment, control, and curiosity, which follow closely with Griffiths' biopsychosocial model of addiction.

Another relevant theory, the uses and gratifications theory (UGT), "helps understand background characteristics and individual differences motivating people to choose using particular types of mass media. UGT can explain how people with certain types of psychological and/or demographic characteristics may be drawn to increasingly use specific types of smartphone features" to achieve gratification [63]. Why are some people more prone to using Instagram and obsessing over how many "likes" they have accrued on their posts? What is it about some people having to constantly check their Twitter to see if their "tweets" are trending or how many "retweets" they have received? This theory could play a role in defining and characterizing social media addiction as a subset of SA.

\subsection{Group-Specific Variation in SA}

Of the total 108 studies, 84 (78\%) focused on adolescents or emerging adults (ranging from elementary, middle, and high school students (32 of 84 studies, $38 \%$ ) to college/university students (50 of $84,59 \%$ ) (the remaining two studies looked at both high school and university students)) and mentioned that smartphone use was highly prevalent among this youth/young adult group as compared to a more elderly group. Fourteen studies (13\% of 108) focused on adults, and 10 studies included both child and adult participants, usually family members (10\% of 108$)$ (refer to Table 4 ).

Nineteen studies (18\%) reported that gender was a significant predictor of SA, specifically that being female was significantly associated with higher tendencies toward SA or smartphone dependency or that SA/dependency/risk for SA was more prevalent in females than in males. Sixteen studies $(15 \%)$ suggested that females may be more likely to be addicted to social media while males may be more 
likely to be addicted to gaming. However, no consistent findings regarding the subtypes of SA by gender were uncovered.

\subsection{Generalizability of Study Results and Biases}

Across all studies (refer to Table 4), the average percent of male participants was $46 \%$ (SD $=13$ ). Twenty-four countries were represented in this review, with 90 studies (83\%) categorized as a South, Southeast, or East Asian country (in order of frequency: South Korea, China, Turkey, Taiwan, India, Saudi Arabia, Lebanon, Bangladesh, Hong Kong, Indonesia, Iran, Iraq, and Malaysia).

All studies reported gender and age, and seven reported ethnicity (six studies with American adult participants and one with Brazilian adult participants). We also considered other common sociodemographic characteristics (SDCs) measures, including biometrics (e.g., height, weight, and BMI); number of children; education, income, marital, and work/employment status; and region (northern, southern, western, or eastern) or residence (urban or rural). Fifty of the 108 studies (46\%) did not measure any SDCs, $27(25 \%)$ looked at 1 SDC, $18(17 \%)$ looked at 2, $9(8 \%)$ looked at 3, and $4(4 \%)$ looked at 4 SDCs.

About a third of the studies (35\%) reported sampling bias (from convenience sampling), which did not allow for generalization of the findings outside of the study group population. For example, the results from studies focusing on university students may not apply to the general public. Recall bias of participants' self-reports on actual smartphone use and/or dependence, information bias, social desirability bias, response bias, and selection bias were also noted. Thirty-nine of the studies (36\%) also reported multiple biases while 17 (16\%) did not report any biases.

Only two studies used longitudinal data [64,65], and none of the others conducted follow-up assessments on the cross-sectional analyses of smartphone use and behavior. That is, limitations included: The use of cross-sectional data, which limits the ability to draw causal inferences, especially when determining the direction of association between SA/PSU and risk factors of interest; small sample size; not being able to determine whether study characteristics preceded SA/PSU development or were the outcome of smartphone use; use of less-than-optimal instruments tending to be subjective rather than objective; incentive-influenced survey answers; and attrition. The studies that did not report biases or limitations may have overlooked noting the potential ones mentioned above.

Nearly all of the authors recommended one or more of the following: Conducting future studies to further investigate the relationship between SA/PSU and related health risks, thoroughly identifying positive and negative outcomes, conducting longitudinally designed research studies with broader sample profiles; creating public health educational programs to inform the public of the physical and psychological risks associated with SA/PSU, and developing proper evidence-based strategies and interventions to address SA/PSU.

\subsection{Quality of Samples and Level of Evidence}

Examining data collection methods (refer to Table 5), about a half of the studies (49\%) reported using convenience sampling, about a third (34\%) reported random sampling, and the rest $(17 \%)$ did not clearly report a sampling method. Most of the studies (90\%) did not calculate an appropriate sample size before recruiting participants. In terms of sample sizes (with a range of 52-7003), 17 studies (16\%) had 200 participants or less, 41 (38\%) fell in the 201-500 range, $24(22 \%)$ in the 501-1000 range, and 26 (24\%) had more than 1001 participants.

In terms of associations with SA/PSU, 1 study focused on academic data, $11(10 \%)$ presented physiological data (e.g., craniovertebral angle, skin conductance), 40 (37\%) psychological data (e.g., depression, anxiety), 16 (15\%) social data (e.g., social connectedness, alexithymia), $8(7 \%)$ smartphone usage data (e.g., duration, types of functions used), and $28(26 \%)$ reported a mix of those categories.

Most of the studies were subjective reports (90\%). Ten of the 11 objective reports (one academic, four physiological, one psychological, two social, and two smartphone usage) were deemed suggestive 
(but not sufficient or indicative) of a causal association with SA/PSU. The remaining objective report (psychological) was deemed insufficient because the link between SA and national identity was not clear to us. Seven of the 97 subjective reports (six social, one psychological) were deemed suggestive (but not sufficient or indicative) because unlike other psychological reports (within which subjective reports can be verified with objective testing, or physiological measures), certain social aspects (e.g., loneliness, family history of alcohol addiction, need for social assurance, life satisfaction, parental neglect, friends' support, and peer relationships) are hard to measure with objective testing and so subjective data is helpful and could be relatively valid and reliable in those cases.

\section{Discussion}

\subsection{Smartphone Addiction on a Continuum of Addictive Behaviors}

\subsubsection{Is Smartphone Addiction Really an Entity of Its Own?}

With smartphone use engrained as the social norm today, and the pervasive use ever increasing despite an awareness of the health risks and adverse consequences, now the question is if excessive smartphone use is truly an addiction (SA) or just problematic smartphone use (PSU). This review highlights that SA articles already assume that SA is an addiction and frame the research as such, while PSU articles explore reasons why PSU falls short of meeting the necessary criteria to be considered a true addiction. The growing literature on excessive smartphone use has conceptualized the disorder as an addictive behavior. Specifically, advocates of PSU argue that the ethiopathological pathways and processes have not yet been identified in SA research, suggesting that SA interventions are simply targeting the symptoms rather than the underlying causes [66]. We agree that research on SA etiology is necessary in order for the disorder to be properly and accurately diagnosed. Although that will take a considerable amount of time and effort to accrue, hopefully our future capabilities to capture sound psychosocial as well as neurobiological evidence will be established. There has also been criticism that the only support to date of SA being an addiction is limited to "exploratory studies relying on self-report data which is collected via convenience sampl[ing]" [4]. Although the majority of recent reports have been subjective, there have been attempts to collect objective data that are promising, especially if those studies are planning on following up on the data in the future. It seems more studies are also trying to carry out randomized studies with larger sample sizes in order to expand on the empirical data available in the field of SA research.

The current review found that all of the assessment scales measuring SA/PSU met at least half of Griffiths' criteria, which need to be met in order for a disorder to be considered an addiction, according to Griffiths. However, PSU advocates counter that conforming excessive smartphone use within addiction models, such as Griffiths', could oversimplify the disorder and result in clinical irrelevance [66]. Specifically, attempts at conceptualizing tolerance with respect to SA may be insufficient, as inferring tolerance based on the increasing use of the smartphone could vary by several factors, such as age (e.g., teens pressured by peers to participate in social media use), subscription status (subscriptions to apps are paid in full or need to be paid monthly), relationship status (single versus in a relationship), occupation status (student, works desk job), and significant life events (starting or ending a romantic relationship) [4]. Although it could indeed oversimply the condition, Griffiths' model has been widely used as a biopsychosocial framework to operationalize addictive components, and so it is a good starting point to conceptualize the level of addiction to the smartphone. Also, once again, in order to truly verify conceptualizations like tolerance, there is a need for neurobiological evidence (e.g., "alteration/sensitization in specific cerebral circuitries" [4]) to confirm tolerance levels increasing in a smartphone user.

We considered using the "Surgeon General's criteria" (the "Hill criteria") for causality, noting the: (1) Consistency, (2) strength, (3) specificity, (4) temporal relationship, and (5) coherence of the association between SA/PSU and the variables of interest. However, we chose not to include consistency, as none of the studies conducted follow-up studies in different populations under different circumstances, 
or specificity, which researchers have criticized to be useless or misleading [67]. However, the vast majority of the studies only met the coherence criteria. Additionally, since all but two studies were longitudinal, temporality could not be assessed. Future studies could consider addressing the consistency, strength, and temporal relationship of the association by conducting longitudinal and follow-up studies, with adequate sample sizes ensuring power in the analyses.

\subsubsection{Assuming SA Is an Addiction, Is It an Addiction to the Device or on the Device?}

What used to be a novelty-type activity about a decade ago has now become more of a normative behavior [68]; excessive smartphone use is one of the more recent forms of human-machine interaction, raising public health alarms. However, the current review notes that despite the growing body of research, many studies still do not clearly define SA. In fact, in some of the literature, SA and PSU are still interchangeable terms. To add to the ambiguity, only about one in five articles make the distinction that $\mathrm{SA}$ is an addiction specifically to the mobile features provided by the smartphone (texting; various social media apps, such as Twitter and Instagram) that desktops and even laptops cannot match in terms of ease of portability and handheld capabilities. The vast majority of recent articles did not make a clear distinction of SA from related addictions, such as Internet or gaming addiction, suggesting that researchers may assume that SA is actually a subset of technological addictions. Those articles focused more on the mediating role of associated variables (e.g., emotional intelligence and coping style [48]) on SA and/or the moderating role of variables on the relation between risk factors of SA and SA (e.g., whether perceived social support and depression would moderate the relationship between sensation seeking and adolescent SA [49]) rather than differentiating SA from other technological addictions or specifying that SA is an addiction distinct from other addictions (e.g., gaming, gambling, shopping, socializing, sex) based on what the smartphone offers users.

We believe that the only difference between smartphone and Internet addiction (IA) is that SA is essentially IA presented through a highly portable device. IA is a potential addiction restricted to a stationary type of device. In contrast, excessive smartphone use is more prevalent due to its ease of access and portability, and the smartphone itself has become the most common device of choice for people today to access the Internet. Currently, the smartphone is the medium of problematic overuse of apps, games, and social networking site interactions. In the future, perhaps a new behavioral addiction will be virtual reality addiction through use of high-tech contact lens-the ever-changing and improving technological advancements will make possibilities (and potential problems) endless. However, for today, we argue that SA is an emerging addiction to the smartphone content specifically through the smartphone device.

\subsubsection{Generalizability of Results and At-Risk Populations}

The emergence of SA as an addiction is highlighted by the fact that people from various countries are affected by SA, which is not localized to just one continent but worldwide. Although several factors (e.g., not all sociodemographic characteristics of samples being measured, biases, cross-sectional limitations of inferencing causality, insufficient sample sizes, inability to determine directionality of SA and its risk factors, attrition) did not allow for generalization of the findings outside of the study group population, the results still suggested that children and young adults are more affected by SA than other age groups and that men and women are affected by SA in different ways.

In terms of SA affecting youth, one study reported that those at risk of developing SA displayed more severe levels of behavioral and emotional problems, lower self-esteem, and poorer quality of communication with their parents compared with those at normal risk [45]. In terms of gender differences, one study observed that girls who frequently use their smartphones may have a greater tendency to use social networking apps (e.g., Facebook) to upload pictures/share their lives online and therefore have a higher degree of smartphone attachment as compared to boys. Girls have also been reported to form and maintain social relationships and be engaged emotionally through constant app connection while boys mostly use smartphones to communicate through texts [36]. However, not all 
people at risk fall neatly into those observed categories. Implications of these differences require further research among similar group populations (e.g., youths, females versus males, students) around the world to better understand age, gender, and cultural variations in SA that may exist, which could help guide future tailored interventions.

\subsection{Limitations}

For this review, we narrowed our article pool by searching for only "smartphone"|"mobile phone" and "addiction"/"problematic use" terms. We did not actively search for "android", "iPhone", "cell phone", "cellular device", "compulsive use", "phubbing", "snubbing", and "nomophobia", which could have potentially made us miss articles relevant to this review. However, we were able to locate a few relevant articles after reviewing the reference sections of selected articles. Much more research is needed that investigates differences as a function of demographic variables, such as gender, age, or ethnicity. Another limitation is that our interpretations of categorizing certain variables into their respective groupings may not match others' opinions. For example, while we may propose that a certain subjective report is suggestive of a causal relationship with SA/PSU, another researcher may disagree and say that it is insufficient evidence. Another example would be missing a guiding theory used in articles, making Table 3 incomplete.

\section{Conclusions}

Most studies to date seem to assume that SA is a valid behavioral addiction, many forming their assumptions based on Griffiths' component model of addiction, and framing their study based on that assumption. The interchangeable use of the terms SA and PSU, inconsistent methodological approaches used to study SA (e.g., varied use of SA/PSU scales among research), lack of standardized diagnostic criteria, and unclear distinctions of SA from other related addictions make it difficult to make a conclusive statement on the status of SA, which could be considered "an ill-defined and heterogeneous construct" [67]. With no unifying theory on SA, all theories mentioned in the current research highlight the complexity of SA: One theory cannot simply explain SA, but rather several theories and models possibly need to be integrated to better explain its distinct addictive traits in this new technologically advanced era. Much more research is needed to confirm the uniqueness of SA, which encompasses the addictive activities engaged in on the smartphone, which includes apps that are not available on other devices. It is most plausible, based on the current studies, to infer that SA falls on a continuum of additive behaviors, from mild PSU to more extreme addictive behavior, where the consequences need to be addressed, prevented, and potentially treated before the adverse health effects debilitate the smartphone user.

Author Contributions: Conceptualization, methodology, analysis, writing-original draft preparation, S.Y.; writing-Review and editing, supervision, S.S. All authors have read and agreed to the published version of the manuscript.

Funding: This research received no external funding.

Acknowledgments: We would like to thank Dmitri Rozgonjuk and Regina van den Eijnden for their support on this paper.

Conflicts of Interest: The authors declare no conflict of interest.

\section{References}

1. Elhai, J.D.; Dvorak, R.D.; Levine, J.C.; Hall, B.J. Problematic smartphone use: A conceptual overview and systematic review of relations with anxiety and depression psychopathology. J. Affect. Disord. 2017, 207, 251-259. [CrossRef] [PubMed]

2. Sapacz, M.; Rockman, G.; Clark, J. Are we addicted to our cell phones? Comput. Hum. Behav. 2016, 57, 153-159. [CrossRef] 
3. Bianchi, A.; Phillips, J.G. Psychological predictors of problem mobile phone use. Cyberpsychol. Behav. 2005, 8, 39-51. [CrossRef] [PubMed]

4. Billieux, J.; Maurage, P.; Lopez-Fernandez, O.; Kuss, D.J.; Griffiths, M.D. Can Disordered Mobile Phone Use Be Considered a Behavioral Addiction? An Update on Current Evidence and a Comprehensive Model for Future Research. Curr. Addict. Rep. 2015, 2, 156-162. [CrossRef]

5. Choi, S.W.; Kim, D.J.; Choi, J.S.; Ahn, H.; Choi, E.J.; Song, W.Y.; Kim, S.; Youn, H. Comparison of risk and protective factors associated with smartphone addiction and Internet addiction. J. Behav. Addict. 2015, 4, 308-314. [CrossRef]

6. American Psychiatric Association. Diagnostic and Statistical Manual of Mental Disorders, 5th ed.; American Psychiatric Association: Arlington, VA, USA, 2013.

7. ICD-11 for Mortality and Morbidity Statistics. Available online: https://icd.who.int/browse11/1-m/en (accessed on 1 October 2019).

8. Sussman, S. Substance and Behavioral Addictions: Concepts, Causes, and Cures, 1st ed.; Cambridge University Press: Cambridge, UK, 2017.

9. Sussman, S.; Sussman, A.N. Considering the Definition of Addiction. Int. J. Environ. Res. Public Health 2011, 8, 4025-4038. [CrossRef]

10. Panova, T.; Carbonell, X. Is smartphone addiction really an addiction? J. Behav. Addict. 2018, 7, $252-259$. [CrossRef]

11. Sussman, S.; Rozgonjuk, D.; van den Eijnden, R.J.J.M. Substance and behavioral addictions may share a similar underlying process of dysregulation. Addiction 2017, 112, 1717-1718. [CrossRef]

12. Alhassan, A.A.; Alqadhib, E.M.; Taha, N.W.; Alahmari, R.A.; Salam, M.; Almutairi, A.F. The relationship between addiction to smartphone usage and depression among adults: A cross sectional study. BMC Psychiatry 2018, 18, 148. [CrossRef]

13. Chen, C.; Zhang, K.; Gong, X.; Zhao, S.; Lee, M.; Liang, L. Examining the effects of motives and gender differences on smartphone addiction. Comput. Hum. Behav. 2017, 75, 891-902. [CrossRef]

14. Chun, J.S. Conceptualizing effective interventions for smartphone addiction among Korean female adolescents. Child. Youth Serv. Rev. 2018, 84, 35-39. [CrossRef]

15. Elserty, N.S.; Helmy, N.A.; Mounir, K.M. Smartphone addiction and its relation to musculoskeletal pain in Egyptian physical therapy students. Eur. J. Physiother. 2018, 1-9. [CrossRef]

16. Hao, Z.; Jin, L.; Li, Y.; Akram, H.; Saeed, M.F.; Ma, J.; Ma, H.; Huang, J. Alexithymia and mobile phone addiction in Chinese undergraduate students: The roles of mobile phone use patterns. Comput. Hum. Behav. 2019, 97, 51-59. [CrossRef]

17. Jeong, Y.J.; Suh, B.; Gweon, G. Is smartphone addiction different from Internet addiction? comparison of addiction-risk factors among adolescents. Behav. Inf. Technol. 2019, 1-16. [CrossRef]

18. Jin, Y.; Sun, C.; An, J.; Li, J. Attachment Styles and Smartphone Addiction in Chinese College Students: The Mediating Roles of Dysfunctional Attitudes and Self-Esteem. Int. J. Ment. Health Addict. 2017, 15, 1122-1134. [CrossRef]

19. Jo, H.; Na, E.; Kim, D.J. The relationship between smartphone addiction predisposition and impulsivity among Korean smartphone users. Addict. Res. Theory 2017, 26, 1-8. [CrossRef]

20. Konan, N.; Çelik, O.T. The Mediator Role of Interaction Anxiety in the Relationship between Social Support Perception and Smartphone Addiction. J. Educ. Future 2019, 15, 63-75.

21. Kwan, H.; Leung, M. The Structural Model in Parenting Style, Attachment Style, Self-regulation and Self-esteem for Smartphone Addiction. J. Psychol. Behav. Sci. 2017, 3, 85-103. [CrossRef]

22. Sekhon, A. Gender differences in mobile phone addiction and its association with stress among medical students. Indian J. Health Wellbeing 2018, 9, 232-234.

23. Yang, Z.; Asbury, K.; Griffiths, M.D. An Exploration of Problematic Smartphone Use among Chinese University Students: Associations with Academic Anxiety, Academic Procrastination, Self-Regulation and Subjective Wellbeing. Int. J. Ment. Health Addict. 2019, 17, 596-614. [CrossRef]

24. Arnavut, A.; Nuri, C.; Direktör, C. Examination of the relationship between phone usage and smartphone addiction based on certain variables. An. Psicol. 2018, 34, 446-450. [CrossRef]

25. Barnes, S.; Pressey, A.; Scornavacca, E. Mobile Ubiquity: Understanding the Impact of Cognitive Absorption on Smartphone Addiction. Comput. Hum. Behav. 2018, 90, 246-258. [CrossRef] 
26. Chen, B.; Liu, F.; Ding, S.; Ying, X.; Wang, L.; Wen, Y. Gender differences in factors associated with smartphone addiction: A cross-sectional study among medical college students. BMC Psychiatry 2017, 17, 1-9. [CrossRef] [PubMed]

27. Chiang, J.T.; Chang, F.C.; Lee, K.W.; Hsu, S.Y. Transitions in smartphone addiction proneness among children: The effect of gender and use patterns. PLoS ONE 2019, 14, 1-12. [CrossRef]

28. Chung, J.E.; Choi, S.A.; Kim, K.T.; Yee, J.; Kim, J.H.; Seong, J.W.; Seong, J.M.; Kim, J.Y.; Lee, K.E.; Gwak, H.S. Smartphone addiction risk and daytime sleepiness in Korean adolescents: Smartphone addiction and sleep problems. J. Paediatr. Child Health 2018, 54, 800-806. [CrossRef]

29. Duke, E.; Montag, C. Smartphone addiction, daily interruptions and self-reported productivity. Addict. Behav. Rep. 2017, 19, 90-95. [CrossRef]

30. Kim, S.G.; Park, J.; Kim, H.T.; Pan, Z.; Lee, Y.; McIntyre, R.S. The relationship between smartphone addiction and symptoms of depression, anxiety, and attention-deficit/hyperactivity in South Korean adolescents. Ann. Gen. Psychiatry 2019, 9, 1-8. [CrossRef]

31. Kuang-Tsan, C.; Fu-Yuan, H. Study on Relationship Among University Students' Life Stress, Smart Mobile Phone Addiction, and Life Satisfaction. J. Adult Dev. 2017, 24, 109-118. [CrossRef]

32. Kuss, D.J.; Kanjo, E.; Crook-Rumsey, M.; Kibowski, F.; Wang, G.Y.; Sumich, A. Problematic Mobile Phone Use and Addiction Across Generations: The Roles of Psychopathological Symptoms and Smartphone Use. J. Technol. Behav. Sci. 2018, 3, 141-149. [CrossRef]

33. Lee, H.; Kim, J.W.; Choi, T.Y. Risk Factors for Smartphone Addiction in Korean Adolescents: Smartphone Use Patterns. J. Korean Med. Sci. 2017, 32, 1674-1679. [CrossRef]

34. Lee, C.; Lee, S.J. Prevalence and predictors of smartphone addiction proneness among Korean adolescents. Child. Youth Serv. Rev. 2017, 77, 10-17. [CrossRef]

35. Nayak, J.K. Relationship among smartphone usage, addiction, academic performance and the moderating role of gender: A study of higher education students in India. Comput. Educ. 2018, 123, 164-173. [CrossRef]

36. Yang, S.Y.; Lin, C.Y.; Huang, Y.C.; Chang, J.H. Gender differences in the association of smartphone use with the vitality and mental health of adolescent students. J. Am. Coll. Health 2018, 66, 693-701. [CrossRef] [PubMed]

37. Abed, S.N.; Abd, R.K.; Salim, I.D.; Jamal, N.A.R. Health problems of Mobile Phone Addiction for sample of students and their health awareness at institute technical of kut. J. Pharm. Sci. Res. 2018, 10, 412-415.

38. Alavi, S.S.; Ghanizadeh, M.; Mohammadi, M.R.; Kalhory, S.M.; Jannatifard, F.; Sepahbodi, G. The Survey personal and national identity between individual with cell phone addiction disorder and normal smartphone users. Iran. J. Psychiatry 2018, 13, 15-21.

39. Chen, C.Y. Smartphone addiction: Psychological and social factors predict the use and abuse of a social mobile application. Inf. Commun. Soc. 2018, 1-14. [CrossRef]

40. Kim, J.H. Smartphone-mediated communication vs. face-to-face interaction: Two routes to social support and problematic use of smartphone. Comput. Hum. Behav. 2017, 67, 282-291. [CrossRef]

41. Kim, H.J.; Min, J.Y.; Min, K.B.; Lee, T.J.; Yoo, S. Relationship among family environment, self-control, friendship quality, and adolescents' smartphone addiction in South Korea: Findings from nationwide data. PLoS ONE 2018, 13, 1-13. [CrossRef]

42. Kim, Y.; Lee, N.; Lim, Y. Gender differences in the association of smartphone addiction with food group consumption among Korean adolescents. Public Health 2017, 145, 132-135. [CrossRef]

43. Kumcagiz, H. Quality of Life as a Predictor of Smartphone Addiction Risk Among Adolescents. Technol. Knowl. Learn. 2019, 24, 117-127. [CrossRef]

44. Lee, J.E.; Jang, S.I.; Ju, Y.J.; Kim, W.; Lee, H.J.; Park, E.C. Relationship between Mobile Phone Addiction and the Incidence of Poor and Short Sleep among Korean Adolescents: A Longitudinal Study of the Korean Children \& Youth Panel Survey. J. Korean Med. Sci. 2017, 32, 1166-1172. [CrossRef] [PubMed]

45. Lee, J.; Sung, M.J.; Song, S.H.; Lee, Y.M.; Lee, J.J.; Cho, S.M.; Park, M.K.; Shin, Y.M. Psychological Factors Associated with Smartphone Addiction in South Korean Adolescents. J. Early Adolesc. 2018, 38, 288-302. [CrossRef]

46. Mahapatra, S. Smartphone addiction and associated consequences: Role of loneliness and self-regulation. Behav. Inf. Technol 2019, 38, 833-844. [CrossRef] 
47. Sok, S.R.; Seong, M.H.; Ryu, M.H. Differences of Self-Control, Daily Life Stress, and Communication Skills between Smartphone Addiction Risk Group and General Group in Korean Nursing Students. Psychiatr. Q 2019, 90, 1-9. [CrossRef] [PubMed]

48. Sun, J.; Liu, Q.; Yu, S. Child neglect, psychological abuse and smartphone addictionamong Chinese adolescents: The roles of emotional intelligence and coping style. Comput. Hum. Behav. 2019, 90, 74-83. [CrossRef]

49. Wang, P.; Lei, L.; Wang, X.; Nie, J.; Chu, X.; Jin, S. The exacerbating role of perceived social support and the "buffering" role of depression in the relation between sensation seeking and adolescent smartphone addiction. Personal. Indivd Differ. 2018, 130, 129-134. [CrossRef]

50. Wang, P.; Zhao, M.; Wang, X.; Xie, X.; Wang, Y.; Lei, L. Peer relationship and adolescent smartphone addiction: The mediating role of self-esteem and the moderating role of the need to belong. J. Behav. Addict. 2017, 6, 708-717. [CrossRef]

51. Xu, T.T.; Wang, H.Z.; Fonseca, W.; Zimmerman, M.A.; Rost, D.H.; Gaskin, J.; Wang, J.L. The relationship between academic stress and adolescents' problematic smartphone usage. Addict. Res. Theory 2019, 27, 162-169. [CrossRef]

52. Yang, X.; Zhou, Z.; Liu, Q.; Fan, C. Mobile Phone Addiction and Adolescents' Anxiety and Depression: The Moderating Role of Mindfulness. J. Child Fam. Stud. 2019, 28, 822-830. [CrossRef]

53. Yildiz Durak, H. Investigation of nomophobia and smartphone addiction predictors among adolescents in Turkey: Demographic variables and academic performance. Soc. Sci. J. 2018, in press. [CrossRef]

54. Yild1z, M. Emotion regulation strategies as predictors of internet addiction and smartphone addiction in adolescents. J Educ. Sci. Psychol. 2017, 7, 66-78.

55. Youn, H.; Lee, S.I.; Lee, S.H.; Kim, J.Y.; Kim, J.H.; Park, E.J.; Park, J.S.; Bhang, S.Y.; Lee, M.S.; Lee, Y.J.; et al. Exploring the Differences between Adolescents' and Parents' Ratings on Adolescents' Smartphone Addiction. J. Korean Med. Sci. 2018, 33, 1-11. [CrossRef] [PubMed]

56. Moher, D.; Liberati, A.; Tetzlaff, J.; Altman, D.G.; PRISMA Group. Preferred reporting items for systematic reviews and meta-analyses: The PRISMA statement. PLoS Med. 2009, 6, 1-6. [CrossRef]

57. Griffiths, M. A 'components' model of addiction within a biopsychosocial framework. J. Subst. Use 2005, 10, 191-197. [CrossRef]

58. Office of the Surgeon General (US); Office on Smoking and Health (US). The Health Consequences of Smoking: A Report of the Surgeon General; Centers for Disease Control and Prevention (US): Atlanta, GA, USA, 2004.

59. Kwon, M.; Lee, J.Y.; Won, W.Y.; Park, J.W.; Min, J.A.; Hahn, C.; Gu, X.Y.; Choi, J.H.; Kim, D.J. Development and Validation of a Smartphone Addiction Scale (SAS). PLOS ONE 2013, 8, 1-7. [CrossRef] [PubMed]

60. Lin, Y.H.; Chang, L.R.; Lee, Y.H.; Tseng, H.W.; Kuo, T.B.; Chen, S.H. Development and validation of the Smartphone Addiction Inventory (SPAI). PLoS ONE 2014, 9, 1-5. [CrossRef] [PubMed]

61. Kim, J.H. Psychological issues and problematic use of smartphone: ADHD's moderating role in the associations among loneliness, need for social assurance, need for immediate connection, and problematic use of smartphone. Comput. Hum. Behav. 2018, 80, 390-398. [CrossRef]

62. Cocoradă, E.; Maican, C.I.; Cazan, A.M.; Maican, M.A. Assessing the smartphone addiction risk and its associations with personality traits among adolescents. Child. Youth Serv. Rev. 2018, 93, 345-354. [CrossRef]

63. Elhai, J.D.; Levine, J.C.; Dvorak, R.D.; Hall, B.J. Non-social features of smartphone use are most related to depression, anxiety and problematic smartphone use. Comput. Hum. Behav. 2017, 69, 75-82. [CrossRef]

64. Herrero, J.; Torres, A.; Vivas, P.; Urueña, A. Smartphone Addiction and Social Support: A Three-year Longitudinal Study. Psychosoc. Interv. 2019, 28, 111-118. [CrossRef]

65. Herrero, J.; Urueña, A.; Torres, A.; Hidalgo, A. Socially Connected but Still Isolated: Smartphone Addiction Decreases Social Support Over Time. Soc. Sci. Comput. Rev. 2017, 37, 73-88. [CrossRef]

66. Billieux, J.; Philippot, P.; Schmid, C.; Maurage, P.; De Mol, J.; Van der Linden, M. Is Dysfunctional Use of the Mobile Phone a Behavioural Addiction? Confronting Symptom-Based Versus Process-Based Approaches. Clin. Psychol. Psychother. 2015, 22, 460-468. [CrossRef] [PubMed] 
67. Rothman, K.J.; Greenland, S. Hill's Criteria for Causality. Encyclopedia of Biostatistics, 2nd ed; John Wiley \& Sons, Ltd.: Hoboken, NJ, USA, 2005. [CrossRef]

68. Pinna, F.; Dell'Osso, B.; Di Nicola, M.; Janiri, L.; Altamura, A.C.; Carpiniello, B.; Hollander, E. Behavioural addictions and the transition from DSM-IV-TR to DSM-5. J. Psychopathol. 2015, 21, 380-389.

(C) 2020 by the authors. Licensee MDPI, Basel, Switzerland. This article is an open access article distributed under the terms and conditions of the Creative Commons Attribution (CC BY) license (http://creativecommons.org/licenses/by/4.0/). 